TRANSACTIONS OF THE

AMERICAN MATHEMATICAL SOCIETY

Volume 358, Number 11, November 2006, Pages 4959-4980

S 0002-9947(06)03876-1

Article electronically published on April 11, 2006

\title{
OPEN LOCI OF GRADED MODULES
}

\author{
CHRISTEL ROTTHAUS AND LIANA M. ŞEGA
}

\begin{abstract}
Let $A=\bigoplus_{i \in \mathbb{N}} A_{i}$ be an excellent homogeneous Noetherian graded ring and let $M=\bigoplus_{n \in \mathbb{Z}} M_{n}$ be a finitely generated graded $A$-module. We consider $M$ as a module over $A_{0}$ and show that the $\left(S_{k}\right)$-loci of $M$ are open in $\operatorname{Spec}\left(A_{0}\right)$. In particular, the Cohen-Macaulay locus $U_{C M}^{0}=\left\{\mathfrak{p} \in \operatorname{Spec}\left(A_{0}\right) \mid\right.$ $M_{\mathfrak{p}}$ is Cohen-Macaulay\} is an open subset of $\operatorname{Spec}\left(A_{0}\right)$. We also show that the $\left(S_{k}\right)$-loci on the homogeneous parts $M_{n}$ of $M$ are eventually stable. As an application we obtain that for a finitely generated Cohen-Macaulay module $M$ over an excellent ring $A$ and for an ideal $I \subseteq A$ which is not contained in any minimal prime of $M$, the $\left(S_{k}\right)$-loci for the modules $M / I^{n} M$ are eventually stable.
\end{abstract}

\section{INTRODUCTION}

A well-known theorem of Grothendieck states that if $M$ is a finitely generated module over an excellent Noetherian ring $A$, then for all $k \in \mathbb{N}$ the $\left(S_{k}\right)$-locus of $M$

$$
U_{S_{k}}(M)=\left\{\mathfrak{p} \in \operatorname{Spec}(A) \mid M_{\mathfrak{p}} \text { satisfies }\left(S_{k}\right)\right\}
$$

is an open subset of $\operatorname{Spec}(A)$. As usual, $\left(S_{k}\right)$ denotes the Serre condition, that is, $M_{\mathfrak{p}}$ satisfies $\left(S_{k}\right)$ if for all $\mathfrak{q} \in \operatorname{Spec}(A)$ with $\mathfrak{q} \subseteq \mathfrak{p}$ it holds that

$$
\operatorname{depth}_{A_{\mathfrak{q}}}\left(M_{\mathfrak{q}}\right) \geq \min \left(k, \operatorname{dim}\left(M_{\mathfrak{q}}\right)\right) .
$$

It also follows that for such modules $M$ the Cohen-Macaulay locus

$$
U_{C M}(M)=\left\{\mathfrak{p} \in \operatorname{Spec}(A) \mid M_{\mathfrak{p}} \text { is Cohen-Macaulay }\right\}
$$

is an open subset of $\operatorname{Spec}(A)$.

Let $A=\bigoplus_{n>0} A_{n}$ be a Noetherian graded excellent homogeneous ring and $M=\bigoplus_{i \in \mathbb{Z}} M_{i}$ a finitely generated graded $A$-module. Considered as a module over the base ring $A_{0}, M$ is a direct sum of finitely generated $A_{0}$-modules. Moreover, if the base ring $A_{0}$ is local, the standard notion of depth is meaningful for the $A_{0}$-module $M$ and we may consider its $\left(S_{k}\right)$-loci

$$
U_{S_{k}}^{0}(M)=\left\{\mathfrak{p} \in \operatorname{Spec}\left(A_{0}\right) \mid M_{\mathfrak{p}} \text { satisfies } S_{k}\right\},
$$

where $M_{\mathfrak{p}}$ denotes the localization of $M$ at the multiplicative set $A_{0} \backslash \mathfrak{p}$. In this paper we prove that under these assumptions the $\left(S_{k}\right)$-loci of the $A_{0}$-module $M$ are open subsets of $\operatorname{Spec}\left(A_{0}\right)$. In particular, the Cohen-Macaulay locus of $M$ (as

Received by the editors March 23, 2004 and, in revised form, September 28, 2004.

2000 Mathematics Subject Classification. Primary 13A02, 13C15, 13F40; Secondary 13A30, $13 \mathrm{C} 14$.

(C)2006 American Mathematical Society Reverts to public domain 28 years from publication 
an $A_{0}$-module)

$$
U_{C M}^{0}(M)=\left\{\mathfrak{p} \in \operatorname{Spec}\left(A_{0}\right) \mid M_{\mathfrak{p}} \text { is Cohen-Macaulay }\right\}
$$

is an open subset of $\operatorname{Spec}\left(A_{0}\right)$.

The proof follows the main ideas of Grothendieck's proof. It is, however, not merely a copy of the proof in EGA and requires a number of modifications. For the benefit of the reader we have included complete proofs of the results. Our proof is based on the following two observations: First, if $A$ is a polynomial ring over the base ring $A_{0}$, then every graded resolution of $M$ by finitely generated graded free $A$ modules provides a free resolution of the $A_{0}$-module $M$ which is finitely generated on the homogeneous parts. The second is a result by Hochster and Roberts which states for the $A$-module $M$ that there is an element $a \in A_{0} \backslash(0)$ so that $M_{a}$ is a free $\left(A_{0}\right)_{a}$-module provided that the ring $A_{0}$ is a domain.

The paper is organized as follows:

The first section contains basic facts about graded rings and modules which are relevant for the rest of the paper. As a main result we obtain that the AuslanderBuchsbaum formula holds for the $A_{0}$-module $M$.

The second section shows that the codepth-loci of $M$ are open in $\operatorname{Spec}\left(A_{0}\right)$. This is the main step in proving the openness of the $\left(S_{k}\right)$-loci which we present in the next section.

In Section 4 we consider the homogeneous parts of the graded module $M$. We show that the codepth-loci and $\left(S_{k}\right)$-loci of the homogeneous parts of $M$ are eventually stable. This is applied in the last section to the case of a finitely generated module $M$ over an excellent Noetherian ring $A$. If $I \subseteq A$ is an ideal we recover a well-known result by Kodiyalam [7, namely that for $k \geq k_{0}$

$$
\operatorname{depth}\left(M / I^{k} M\right)=\operatorname{depth}\left(M / I^{k_{0}} M\right) .
$$

We also show that if $M$ is a Cohen-Macaulay module over $A$ and if $I \subseteq A$ is not contained in a minimal prime of $M$, then the codepth- and $\left(S_{k}\right)$-loci of $M / I^{n} M$ are eventually stable.

\section{BASIC FACTS}

In this paper we assume that $A=\bigoplus_{i \in \mathbb{N}} A_{i}$ is a Noetherian homogeneous graded ring and that $M=\bigoplus_{i \in \mathbb{Z}} M_{i}$ is a finitely generated $A$-module. As usual, we let $A_{+}$ denote the irrelevant ideal of $A$, that is, $A_{+}=\bigoplus_{i \geq 1} A_{i}$.

If $\mathfrak{p} \in \operatorname{Spec}\left(A_{0}\right)$ is a prime ideal of $A_{0}$, then $M_{\mathfrak{p}}$ denotes the localization $S^{-1} M$ where $S=A_{0} \backslash \mathfrak{p}$. Note that $M_{\mathfrak{p}}$ is a graded module over the graded ring $A_{\mathfrak{p}}$.

Our goal is to show that if $A$ is excellent, then the codepth-loci and the $\left(S_{k}\right)$-loci of $M$, considered as a module over the base ring $A_{0}$, are open subsets of $\operatorname{Spec}\left(A_{0}\right)$.

1.1. General remarks. We begin our investigation with some well-known facts about graded modules. Since these results are frequently used throughout the paper, we include them together with their (short) proofs in this introductory section.

1.1.1. Lemma. There exists an integer $t$ so that $\operatorname{ann}_{A_{0}}\left(M_{t}\right)=\operatorname{ann}_{A_{0}}\left(M_{k}\right)$ for all $k \geq t$. 
Proof. For all $k \in \mathbb{Z}$ set $J_{k}=\operatorname{ann}_{A_{0}}\left(M_{k}\right)$. Since $A$ is homogeneous and $M$ is a finitely generated $A$-module, there exists $t_{0} \in \mathbb{Z}$ such that

$$
A_{1} M_{k}=M_{k+1} \quad \text { for all } \quad k \geq t_{0} .
$$

We conclude $J_{k} \subseteq J_{k+1}$ for all $k \geq t_{0}$. Since $A_{0}$ is Noetherian, there then exists $t \geq t_{0}$ so that $J_{k}=J_{t}$ for all $k \geq t$.

1.1.2. Lemma. The following two functions are well defined and surjective:

(1) The function $\varphi: \operatorname{Supp}_{A}(M) \rightarrow \operatorname{Supp}_{A_{0}}(M)$ defined by $\varphi(P)=P \cap A_{0}$.

(2) The function $\psi: \operatorname{Ass}_{A}(M) \rightarrow \operatorname{Ass}_{A_{0}}(M)$ defined by $\psi(P)=P \cap A_{0}$.

Proof. (1) If $P \in \operatorname{Supp}_{A}(M)$, then $M_{P} \neq 0$ and in particular $M_{\mathfrak{p}} \neq 0$, where $\mathfrak{p}=P \cap A_{0}$. This shows that $\varphi$ is well defined. Let $\mathfrak{p} \in \operatorname{Supp}_{A_{0}}(M)$. Then

$$
M_{\mathfrak{p}}=\bigoplus_{i \in \mathbb{Z}}\left(M_{i}\right)_{\mathfrak{p}} \neq 0
$$

and we may consider $M_{\mathfrak{p}}$ as a graded module over the graded ring $A_{\mathfrak{p}}$. Note that $A_{\mathfrak{p}}$ is a ${ }^{*}$ local ring with unique graded maximal ideal $\mathfrak{m}=\mathfrak{p}\left(A_{0}\right)_{\mathfrak{p}} \oplus\left(A_{+}\right)_{\mathfrak{p}}$. Since all minimal primes of $\operatorname{Supp}_{A_{\mathfrak{p}}}\left(M_{\mathfrak{p}}\right)$ are graded, $\mathfrak{m} \in \operatorname{Supp}_{A_{\mathfrak{p}}}\left(M_{\mathfrak{p}}\right)$. Thus there is a prime $P \in \operatorname{Supp}_{A}(M)$ with $P \cap A_{0}=\mathfrak{p}$.

(2) If $P \in \operatorname{Ass}_{A}(M)$, then there exists $y \in M$ so that $\operatorname{ann}_{A}(y)=P$. Thus $\operatorname{ann}_{A_{0}}(y)=P \cap A_{0}=\mathfrak{p}$ and $\mathfrak{p} \in \operatorname{Ass}_{A_{0}}(M)$. Conversely, let $\mathfrak{p} \in \operatorname{Ass}_{A_{0}}(M)$. Consider again the graded $A_{\mathfrak{p}}$-module $M_{\mathfrak{p}}$. There exists $z \in M_{\mathfrak{p}}$ so that $\operatorname{ann}_{\left(A_{0}\right)_{\mathfrak{p}}}(z)=\mathfrak{p}\left(A_{0}\right)_{\mathfrak{p}}$, and therefore

$$
\mathfrak{p}\left(A_{0}\right)_{\mathfrak{p}} \subseteq \bigcup_{Q \in \operatorname{Ass}_{A_{\mathfrak{p}}}\left(M_{\mathfrak{p}}\right)} Q
$$

Since $M_{\mathfrak{p}}$ is a finitely generated $A_{\mathfrak{p}}$-module, there exists $Q \in \operatorname{Ass}_{A_{\mathfrak{p}}}\left(M_{\mathfrak{p}}\right)$ with $\mathfrak{p}\left(A_{0}\right)_{\mathfrak{p}} \subseteq Q$. Since $A_{\mathfrak{p}}$ is *local with unique graded maximal ideal $\mathfrak{p}\left(A_{0}\right)_{\mathfrak{p}} \oplus\left(A_{+}\right)_{\mathfrak{p}}$, we obtain $Q \cap\left(A_{0}\right)_{\mathfrak{p}}=\mathfrak{p}\left(A_{0}\right)_{\mathfrak{p}}$, and a preimage $P \in \operatorname{Spec}(A)$ of $Q$ is an associated prime of the $A$-module $M$, with $P \cap A_{0}=\mathfrak{p}$.

Lemma 1.1.2 shows in particular that $M$ as an $A_{0}$-module has a finite set of associated primes.

1.1.3. Lemma. Let $A$ and $M$ be as above and set $I=\operatorname{ann}_{A_{0}}(M)$. For any $\mathfrak{p} \in$ $\operatorname{Spec}\left(A_{0}\right)$ the following hold:

(1) If $M_{\mathfrak{p}}=0$, then there is an element $a \in A_{0} \backslash \mathfrak{p}$ with $M_{a}=0$.

(2) $\operatorname{ann}_{\left(A_{0}\right)_{\mathfrak{p}}}\left(M_{\mathfrak{p}}\right)=I\left(A_{0}\right)_{\mathfrak{p}}$.

Proof. (1) This is a basic fact about Noetherian modules using that $M$ is a finitely generated module over $A$ and $A_{0} \backslash \mathfrak{p}$ is a multiplicative subset of $A$.

(2) Obviously, $I\left(A_{0}\right)_{\mathfrak{p}} \subseteq \operatorname{ann}_{\left(A_{0}\right)_{\mathfrak{p}}}\left(M_{\mathfrak{p}}\right)$. Let $x \in \operatorname{ann}_{\left(A_{0}\right)_{\mathfrak{p}}}\left(M_{\mathfrak{p}}\right)$ with $x=\frac{b}{s}$, where $b \in A_{0}$ and $s \in A_{0} \backslash \mathfrak{p}$. Assume that $m_{1}, \ldots, m_{r}$ is a system of generators of the $A$-module $M$. Since $x \frac{m_{i}}{1}=0$ for all $1 \leq i \leq r$ there is an element $t \in A_{0} \backslash \mathfrak{p}$ with $t b m_{i}=0$ for all $1 \leq i \leq r$. We have that $t b \in I$ and hence $x=\frac{b}{s} \in I\left(A_{0}\right)_{\mathfrak{p}}$. 
1.2. The Auslander-Buchsbaum formula. Let $A=\bigoplus_{i>0} A_{i}$ be a graded Noetherian homogeneous ring with $\left(A_{0}, \mathfrak{m}_{0}\right)$ local and let $M=\bigoplus_{i \in \mathbb{Z}} M_{i}$ be a finitely generated $A$-module. Since $M$ is (in general) not finitely generated as an $A_{0^{-}}$ module, we need to verify that the classical definition of $A_{0}$-depth works in the case of a finitely generated graded module. First note that an element $z \in \mathfrak{m}_{0}$ is regular on $M$ if and only if $z$ is regular on $M_{i}$ for all $i \in \mathbb{Z}$ with $M_{i} \neq 0$. Let $x_{1}, \ldots, x_{s} \in \mathfrak{m}_{0}$ and $y_{1}, \ldots, y_{t} \in \mathfrak{m}_{0}$ be two maximal regular $M$-sequences (as an $A_{0}$-module). Then for all $i \in \mathbb{Z}$ with $M_{i} \neq 0$ the two sequences are regular on the $A_{0}$-module $M_{i}$, and the sets

$$
\begin{aligned}
& \operatorname{Ass}_{A_{0}}\left(M /\left(x_{1}, \ldots, x_{s}\right) M\right)=\bigcup_{i \in \mathbb{Z}} \operatorname{Ass}_{A_{0}}\left(M_{i} /\left(x_{1}, \ldots, x_{s}\right) M_{i}\right), \\
& \operatorname{Ass}_{A_{0}}\left(M /\left(y_{1}, \ldots, y_{t}\right) M\right)=\bigcup_{i \in \mathbb{Z}} \operatorname{Ass}_{A_{0}}\left(M_{i} /\left(y_{1}, \ldots, y_{t}\right) M_{i}\right)
\end{aligned}
$$

are finite by Lemma 1.1.2. The maximality of the first sequences yields that there is an $i \in \mathbb{Z}$ with $M_{i} \neq 0$ and $\mathfrak{m}_{0} \in \operatorname{Ass}_{A_{0}}\left(M_{i} /\left(x_{1}, \ldots, x_{s}\right) M_{i}\right)$. Since the second sequence is also regular on $M_{i}$ we have that $t \leq s$. A similar argument shows that $s \leq t$, and we obtain that two maximal regular sequences on $M$ have the same length. Therefore the classical definition of depth is efficient and we put:

1.2.1. Definition. Let $A$ and $M$ be as above with $\left(A_{0}, \mathfrak{m}_{0}\right)$ local. We define the depth of $M$ as an $A_{0}$-module to be the number

$$
\operatorname{depth}_{A_{0}}(M):=\sup \{n \in \mathbb{N} \mid \exists \text { an } M \text {-sequence of length } n\} \text {. }
$$

In general, for a (not necessarily finitely generated) module $M$ over a Noetherian local ring $A$, the depth of $M$ is defined by means of Koszul homology (see [2, Definition 9.1.1]). In our setting, the definition above coincides with the one in [2].

The aim of this section is to prove the Auslander-Buchsbaum theorem for finitely generated graded modules $M$ over *local graded Noetherian rings $A$ when $M$ is considered a module over the base ring $A_{0}$. There is a generalized version of the Auslander-Buchsbaum theorem which applies to our case (see [3, (12.2)] or [6, Theorem (2.1)]). For the convenience of the reader we include a proof of this theorem in the graded case, which only makes use of the classical definition of depth as given above.

1.2.2. Lemma. Let $A$ and $M$ be as above and assume that $\left(A_{0}, \mathfrak{m}_{0}\right)$ is local. Then:

(1) $\operatorname{dim}_{A_{0}}(M)=\sup \left\{\operatorname{dim}_{A_{0}}\left(M_{i}\right) \mid i \in \mathbb{Z}\right\}$

(2) $\operatorname{depth}_{A_{0}}(M)=\inf \left\{\operatorname{depth}_{A_{0}}\left(M_{i}\right) \mid i \in \mathbb{Z}\right.$ with $\left.M_{i} \neq 0\right\}$,

(3) $\operatorname{projdim}_{A_{0}}(M)=\sup \left\{\operatorname{projdim}_{A_{0}}\left(M_{i}\right) \mid i \in \mathbb{Z}\right\}$.

Proof. (1) By Lemma 1.1.1 there is an integer $s \in \mathbb{Z}$ so that $\operatorname{ann}_{A_{0}}\left(M_{k}\right)=\operatorname{ann}_{A_{0}}\left(M_{s}\right)$ for all $k \geq s$. In particular, for all $k \geq s$, $\operatorname{dim}_{A_{0}}\left(M_{k}\right)=\operatorname{dim}_{A_{0}}\left(M_{s}\right)$ and

$$
\operatorname{dim}_{A_{0}}(M)=\operatorname{dim}_{A_{0}}\left(M_{r} \oplus M_{r-1} \oplus \ldots \oplus M_{s-1} \oplus M_{s}\right),
$$

where $r \in \mathbb{Z}$ is the smallest integer $j$ with $M_{j} \neq 0$. The dimension of a finite direct sum of $A_{0}$-modules is the maximum of the dimensions of its summands.

(2) If $r_{1}, \ldots, r_{s} \in A_{0}$ is a regular sequence on $M$, then $r_{1}, \ldots, r_{s}$ is a regular sequence on $M_{i}$ for all $i \in \mathbb{Z}$ with $M_{i} \neq 0$. Thus $\operatorname{depth}_{A_{0}}(M) \leq \operatorname{depth}_{A_{0}}\left(M_{i}\right)$ for all $i \in \mathbb{Z}$ with $M_{i} \neq 0$, and hence

$$
\operatorname{depth}_{A_{0}}(M) \leq \inf \left\{\operatorname{depth}_{A_{0}}\left(M_{i}\right) \mid i \in \mathbb{Z} \text { with } M_{i} \neq 0\right\} .
$$


In order to show the other inequality we proceed by induction on $t=\operatorname{depth}_{A_{0}}(M)$.

Note that by Lemma 1.1.3. $\operatorname{Ass}_{A_{0}}(M)$ is a finite set.

If $t=0$, then $\mathfrak{m}_{0} \in \operatorname{Ass}_{A_{0}}(M)$ and there is an $i \in \mathbb{Z}$ so that $\mathfrak{m}_{0} \in \operatorname{Ass}_{A_{0}}\left(M_{i}\right)$. Thus

$$
\inf \left\{\operatorname{depth}_{A_{0}}\left(M_{i}\right) \mid i \in \mathbb{Z} \text { with } M_{i} \neq 0\right\}=0 .
$$

Now assume that $t=\operatorname{depth}_{A_{0}}(M)>0$. This implies that

Consider an element

$$
\bigcup_{\mathfrak{p} \in \operatorname{Ass}_{A_{0}}(M)} \mathfrak{p} \neq \mathfrak{m}_{0}
$$

$$
r \in \mathfrak{m}_{0} \backslash \bigcup_{\mathfrak{p} \in \operatorname{Ass}_{A_{0}}(M)} \mathfrak{p} .
$$

Since $r$ is regular on $M$, and therefore is regular on $M_{i}$ for all $i \in \mathbb{Z}$ with $M_{i} \neq 0$, we obtain

$$
\operatorname{depth}_{A_{0}}(M / r M)=\operatorname{depth}_{A_{0}}(M)-1,
$$

and for all $i \in \mathbb{Z}$ with $M_{i} \neq 0$,

$$
\operatorname{depth}_{A_{0}}\left(M_{i} / r M_{i}\right)=\operatorname{depth}_{A_{0}}\left(M_{i}\right)-1 .
$$

By the induction hypothesis

$$
\operatorname{depth}_{A_{0}}(M / r M)=\inf \left\{\operatorname{depth}_{A_{0}}\left(M_{i} / r M_{i}\right) \mid i \in \mathbb{Z} \text { and } M_{i} / r M_{i} \neq 0\right\} .
$$

The assertion follows.

(3) For all $i \in \mathbb{Z}$ let $F_{\bullet}^{(i)}$ be a finite free resolution of $M_{i}$. Then

$$
F_{\bullet}=\bigoplus_{i \in \mathbb{Z}} F_{\bullet}^{(i)}
$$

is a free resolution of the $A_{0}$-module $M$ yielding

$$
\operatorname{projdim}_{A_{0}}(M) \leq \sup \left\{\operatorname{projdim}_{A_{0}}\left(M_{i}\right) \mid i \in \mathbb{Z}\right\} .
$$

In order to show the other inequality, assume that $\operatorname{projdim}_{A_{0}}(M)=r$ and consider for all $i \in \mathbb{Z}$ the $r$ th syzygy $T_{r}^{(i)}$ of $M_{i}$ and the exact sequence

$$
0 \longrightarrow T_{r}^{(i)} \longrightarrow F_{r-1}^{(i)} \longrightarrow \ldots \longrightarrow F_{0}^{(i)} \longrightarrow M_{i} \longrightarrow 0 .
$$

By taking direct sums we see that

$$
\bigoplus_{i \in \mathbb{Z}} T_{r}^{(i)}
$$

is an $r$ th syzygy of $M$ and thus projective. Therefore every $T_{r}^{(i)}$ is a projective finitely generated $A_{0}$-module. Since $A_{0}$ is a local Noetherian ring, every $T_{r}^{(i)}$ is a free $A_{0}$-module and thus for all $i \in \mathbb{Z}$

$$
\operatorname{projdim}_{A_{0}}\left(M_{i}\right) \leq r .
$$

This shows (3).

1.2.3. Proposition. Let $A$ and $M$ be as above with $\left(A_{0}, \mathfrak{m}_{0}\right)$ a local ring. Then the Auslander-Buchsbaum formula holds for $M$ as an $A_{0}$-module. That is, if $\operatorname{projdim}_{A_{0}}(M)$ is finite, then

$$
\operatorname{depth}_{A_{0}}(M)+\operatorname{projdim}_{A_{0}}(M)=\operatorname{depth}\left(A_{0}\right) .
$$


Proof. Let projdim $A_{A_{0}}(M)=r<\infty$. Then by Lemma 1.2.2(2) there is an $i \in \mathbb{Z}$ with $\operatorname{projdim}_{A_{0}}(M)=\operatorname{projdim}_{A_{0}}\left(M_{i}\right)$, and for all $j \in \mathbb{Z}$

$$
\operatorname{projdim}_{A_{0}}\left(M_{j}\right) \leq r .
$$

The Auslander-Buchsbaum formula holds for finitely generated $A_{0}$-modules

$$
\operatorname{depth}_{A_{0}}\left(M_{j}\right)+\operatorname{projdim}_{A_{0}}\left(M_{j}\right)=\operatorname{depth}_{A_{0}}\left(A_{0}\right) \text { for all } j \in \mathbb{Z},
$$

and therefore

$$
\operatorname{depth}_{A_{0}}\left(M_{j}\right) \geq \operatorname{depth}_{A_{0}}\left(M_{i}\right) \text { for all } j \in \mathbb{Z} .
$$

Using Lemma 1.2.2(1), we conclude $\operatorname{depth}_{A_{0}}(M)=\operatorname{depth}_{A_{0}}\left(M_{i}\right)$. The AuslanderBuchsbaum formula for $M_{i}$ then gives the desired formula.

\section{Openness OF THE CODEPTH LOCUS}

Throughout this section we assume that $A=\bigoplus_{i \in \mathbb{N}_{0}} A_{i}$ is a graded Noetherian homogeneous ring and that $M=\bigoplus_{i \in \mathbb{Z}} M_{i}$ is a finitely generated $A$-module. Our aim is to generalize and/or modify existing theorems for finitely generated modules over Noetherian rings to the graded case where the module $M$ is considered a module over the base ring $A_{0}$. We begin with a result on the flat locus of the $A_{0}$-module $M$.

2.1. The flat locus of $M$. Our first result is a modification of [8, Theorem 24.3]. The proof follows the proof in Matsumura's book. A key observation is that for a finitely generated graded module $M$ the localizations $M_{\mathfrak{p}}$ are $I$-adically separated for every ideal $I \subseteq\left(A_{0}\right)_{\mathfrak{p}}$.

Proposition. Let $A$ and $M$ be as above. The flat locus of $M$ as an $A_{0}$-module

$$
U^{0}(M)=\left\{\mathfrak{p} \in \operatorname{Spec}\left(A_{0}\right) \mid M_{\mathfrak{p}} \text { is flat over } A_{0}\right\}
$$

is open in $\operatorname{Spec}\left(A_{0}\right)$.

Proof. According to Nagata's criterion on the openness of loci [8, Theorem 24.2] we have to show:

(a) If $\mathfrak{p}, \mathfrak{q} \in \operatorname{Spec}\left(A_{0}\right)$ with $\mathfrak{p} \in U^{0}(M)$ and $\mathfrak{q} \subseteq \mathfrak{p}$, then $\mathfrak{q} \in U^{0}(M)$.

(b) If $\mathfrak{p} \in U^{0}(M)$, then $U^{0}(M)$ contains a nonempty open subset of $V^{0}(\mathfrak{p})=\{\mathfrak{n} \in$ $\left.\operatorname{Spec}\left(A_{0}\right) \mid \mathfrak{p} \subseteq \mathfrak{n}\right\}$.

(a) is trivial. Let $\mathfrak{p} \in U^{0}(M)$, that is, assume that $M_{\mathfrak{p}}$ is flat over $A_{0}$. Set $\bar{A}_{0}=A_{0} / \mathfrak{p}$. By [8, Theorem 22.3] for every $\mathfrak{q} \in V^{0}(\mathfrak{p})$ the module $M_{\mathfrak{q}}$ is flat over $A_{0}$ if and only if $(M / \mathfrak{p} M)_{\mathfrak{q}}$ is flat over $\bar{A}_{0}$ and $\operatorname{Tor}_{1}^{A_{0}}\left(M_{\mathfrak{q}}, \bar{A}_{0}\right)=0$. A similar argument as in the proof of [8, Theorem 23.2] shows that $\operatorname{Tor}_{1}^{A_{0}}\left(M, \bar{A}_{0}\right)$ is a finitely generated module over $A$. Therefore there is an element $a \in A_{0} \backslash \mathfrak{p}$ so that $\left(\operatorname{Tor}_{1}^{A_{0}}\left(M, \bar{A}_{0}\right)\right)_{a}=$ 0 . By applying [8, Theorem 24.1] to the $\bar{A}_{0}$-module $M / \mathfrak{p} M$ we obtain an element $b \in$ $A_{0} \backslash \mathfrak{p}$ so that $(M / \mathfrak{p} M)_{b}$ is a free $\left(\bar{A}_{0}\right)_{b}$-module. Set $D_{a b}^{0}=\left\{\mathfrak{q} \in \operatorname{Spec}\left(A_{0}\right) \mid a b \notin \mathfrak{q}\right\}$. Then for all $\mathfrak{q} \in V^{0}(\mathfrak{p}) \cap D_{a b}^{0}$ we have that $\operatorname{Tor}_{1}^{A_{0}}\left(M_{\mathfrak{q}}, \bar{A}_{0}\right)=0$ and that $(M / \mathfrak{p} M)_{\mathfrak{q}}$ is flat over $\left(\bar{A}_{0}\right)_{\mathfrak{q}}$. Thus by [8, Theorem 22.3] the module $M_{\mathfrak{q}}$ is flat over $\left(A_{0}\right)_{\mathfrak{q}}$ and $M_{\mathfrak{q}}$ is flat over $A_{0}$. 
2.2. A proposition by Auslander. As before, let $A$ be a Noetherian graded homogeneous ring and let $M$ be a finitely generated $A$-module. The following Proposition is an extension of a proposition in EGA [4, (6.11.1) and (6.11.2)] to the (not finitely generated) $A_{0}$-module $M$.

Proposition. The function $\gamma: \operatorname{Spec}\left(A_{0}\right) \longrightarrow \mathbb{N}$ defined by

$$
\gamma(\mathfrak{p})=\operatorname{projdim}_{\left(A_{0}\right)_{\mathfrak{p}}}\left(M_{\mathfrak{p}}\right) \quad \text { for all } \mathfrak{p} \in \operatorname{Spec}\left(A_{0}\right)
$$

is upper semicontinuous. That is, for all $n \in \mathbb{N}$ the set

$$
U_{n}^{0}(M)=\left\{\mathfrak{p} \in \operatorname{Spec}\left(A_{0}\right) \mid \operatorname{projdim}_{\left(A_{0}\right)_{\mathfrak{p}}}\left(M_{\mathfrak{p}}\right) \leq n\right\}
$$

is open in $\operatorname{Spec}\left(A_{0}\right)$.

Proof. Note that the ring $A$ is the homomorphic image of the polynomial $\operatorname{ring} B=$ $A_{0}\left[x_{1}, \ldots, x_{t}\right]$, and that, with the standard grading on the polynomial ring $B$, the graded $B$-module $M$ is finitely generated. We may replace $A$ by $B$ and assume that $A$ is a graded polynomial ring over $A_{0}$. Let $\mathfrak{p} \in \operatorname{Spec}\left(A_{0}\right)$ with $\operatorname{projdim}_{\left(A_{0}\right)_{\mathfrak{p}}}\left(M_{\mathfrak{p}}\right) \leq$ $n$.

Consider a graded finitely generated free resolution of the $A$-module $M$ :

$$
F_{n} \stackrel{\varphi_{n}}{\longrightarrow} F_{n-1} \stackrel{\varphi_{n-1}}{\longrightarrow} \cdots \stackrel{\varphi_{1}}{\longrightarrow} F_{1} \stackrel{\varphi_{0}}{\longrightarrow} M \rightarrow 0,
$$

where the $F_{i}$ are finitely generated graded free $A$-modules and the $\varphi_{i}$ are homogeneous $A$-linear maps. Let $T$ be the $n$th syzygy of $M$, yielding an exact sequence of graded $A$-modules:

$$
0 \rightarrow T \stackrel{\delta}{\longrightarrow} F_{n-1} \stackrel{\varphi_{n-1}}{\longrightarrow} \ldots \stackrel{\varphi_{1}}{\longrightarrow} F_{1} \stackrel{\varphi_{0}}{\longrightarrow} M \rightarrow 0 .
$$

Since all the homogeneous parts of $F_{i}$ are free $A_{0}$-modules and since $T$ is a graded $A$-module, we obtain for all $k \in \mathbb{Z}$ an exact sequence of $A_{0}$-modules

$$
0 \rightarrow T_{k} \stackrel{(\delta)_{k}}{\longrightarrow}\left(F_{n-1}\right)_{k} \stackrel{\left(\varphi_{n-1}\right)_{k}}{\longrightarrow} \ldots \stackrel{\left(\varphi_{1}\right)_{k}}{\longrightarrow}\left(F_{1}\right)_{k} \stackrel{\left(\varphi_{0}\right)_{k}}{\longrightarrow} M_{k} \rightarrow 0
$$

with $\left(F_{i}\right)_{k}$ a finitely generated free $A_{0}$-module. Therefore by considering $(*)$ as an exact sequence of $A_{0}$-modules we obtain that every module $F_{i}$ is free over $A_{0}$ and $T$ is an $n$th syzygy of the $A_{0}$-module $M$. Localization at $\mathfrak{p}$ yields exact sequences:

$$
0 \rightarrow T_{\mathfrak{p}} \stackrel{\delta_{\mathfrak{p}}}{\longrightarrow}\left(F_{n-1}\right)_{\mathfrak{p}} \stackrel{\left(\varphi_{n-1}\right)_{\mathfrak{p}}}{\longrightarrow} \ldots \stackrel{\left(\varphi_{1}\right)_{\mathfrak{p}}}{\longrightarrow}\left(F_{1}\right)_{\mathfrak{p}} \stackrel{\left(\varphi_{0}\right)_{\mathfrak{p}}}{\longrightarrow} M_{\mathfrak{p}} \rightarrow 0 .
$$

Since $\operatorname{projdim}_{\left(A_{0}\right) p}\left(M_{\mathfrak{p}}\right) \leq n$, it follows that $T_{\mathfrak{p}}$ is a projective $\left(A_{0}\right)_{\mathfrak{p}}$-module. Therefore $T_{\mathfrak{p}}$ is a free $\left(A_{0}\right)_{\mathfrak{p}}$-module. Since $T$ is a finitely generated graded $A$-module, it follows from Proposition 2.1 that the set

$$
U^{0}(T)=\left\{\mathfrak{q} \in \operatorname{Spec}\left(A_{0}\right) \mid T_{\mathfrak{q}} \text { is a flat over }\left(A_{0}\right)_{\mathfrak{q}}\right\}
$$

is an open subset of $\operatorname{Spec}\left(A_{0}\right)$. Since $T$ is a finitely generated graded $A$-module,

$$
T=\bigoplus_{i \in \mathbb{Z}} T_{i}
$$

we have for $\mathfrak{q} \in \operatorname{Spec}\left(A_{0}\right)$

$$
T_{\mathfrak{q}}=\bigoplus_{i \in \mathbb{Z}}\left(T_{i}\right)_{\mathfrak{q}}
$$


If $T_{\mathfrak{q}}$ is flat over $\left(A_{0}\right)_{\mathfrak{q}}$, then, by [1, chapter $1, \S 2.3$, Proposition 2], for all $i \in \mathbb{Z}$, $\left(T_{i}\right)_{\mathfrak{q}}$ is flat over $\left(A_{0}\right)_{\mathfrak{q}}$. Since every $\left(T_{i}\right)_{\mathfrak{q}}$ is a finitely generated $\left(A_{0}\right)_{\mathfrak{q}}$-module, each $\left(T_{i}\right)_{\mathfrak{q}}$ is a free $\left(A_{0}\right)_{\mathfrak{q}}$-module and

$$
U^{0}(T)=\left\{\mathfrak{q} \in \operatorname{Spec}\left(A_{0}\right) \mid T_{\mathfrak{q}} \quad \text { is a free over }\left(A_{0}\right)_{\mathfrak{q}}\right\} .
$$

This shows that $\mathfrak{p} \in U^{0}(T)$ and

$$
U^{0}(T) \subseteq\left\{\mathfrak{q} \in \operatorname{Spec}\left(A_{0}\right) \mid \operatorname{projdim}_{\left(A_{0}\right)_{\mathfrak{q}}}\left(M_{\mathfrak{q}}\right) \leq n\right\} .
$$

The set $\left\{\mathfrak{q} \in \operatorname{Spec}\left(A_{0}\right) \mid \operatorname{projdim}_{\left(A_{0}\right)_{\mathfrak{q}}}\left(M_{\mathfrak{q}}\right) \leq n\right\}$ is thus open in $\operatorname{Spec}\left(A_{0}\right)$.

\subsection{A dimension formula.}

Proposition. Let $A$ and $M$ be as above. Assume that $A_{0}$ is catenary and let $\mathfrak{p}$ be a prime ideal in $A_{0}$ with $\mathfrak{p} \in \operatorname{Supp}_{A_{0}}(M)$. Then there is an open subset $U$ in $\operatorname{Spec}\left(A_{0}\right)$ such that $\mathfrak{p} \in U$, and for all $\mathfrak{q} \in U \cap V^{0}(\mathfrak{p})$ we have

$$
\operatorname{dim}\left(M_{\mathfrak{q}}\right)=\operatorname{dim}\left(M_{\mathfrak{p}}\right)+\operatorname{dim}\left(\left(A_{0} / \mathfrak{p}\right)_{\mathfrak{q}}\right) .
$$

Proof. Set $S=A_{0} / \operatorname{ann}_{A_{0}}(M)$ and choose an element $a \in S \backslash \mathfrak{p}$ so that the following equality on the set of minimal primes holds:

$$
\operatorname{Min}\left(S_{\mathfrak{p}}\right)=\operatorname{Min}\left(S_{a}\right) .
$$

Assume that $\operatorname{dim}\left(M_{\mathfrak{p}}\right)=\operatorname{ht}(\mathfrak{p} S)=t$ and choose elements $y_{1}, y_{2}, \ldots, y_{t} \in S$ so that

$$
\begin{gathered}
y_{1} \text { not in a minimal prime of } S_{\mathfrak{p}}, \\
y_{2} \text { not in a minimal prime of } y_{1} S_{\mathfrak{p}}, \\
\ldots \\
y_{t} \text { not in a minimal prime of }\left(y_{1}, \ldots, y_{t-1}\right) S_{\mathfrak{p}} .
\end{gathered}
$$

Then there is an element $b \in S \backslash \mathfrak{p}$ so that

$$
\begin{gathered}
y_{1} \text { not in a minimal prime of } S_{b}, \\
y_{2} \text { not in a minimal prime of } y_{1} S_{b}, \\
\ldots \\
y_{t} \quad \text { not in a minimal prime of }\left(y_{1}, \ldots, y_{t-1}\right) S_{b} .
\end{gathered}
$$

Let $a, b$ also denote preimages of $a$ and $b$ in $A_{0}$ and put $U=D_{a b}=\left\{\mathfrak{q} \in \operatorname{Spec}\left(A_{0}\right) \mid\right.$ $a b \notin \mathfrak{q}\}$. Then for every $\mathfrak{q} \in U \cap V^{0}(\mathfrak{p})$ the elements $y_{1}, \ldots, y_{t}$ extend to a system of parameters of $S_{\mathfrak{q}}$. Since $S_{\mathfrak{p}}$ and $S_{\mathfrak{q}}$ have the same set of minimal primes and since $S$ is catenary, we obtain that

$$
\operatorname{dim}\left(S_{\mathfrak{q}}\right)=\operatorname{dim}\left(S_{\mathfrak{p}}\right)+\operatorname{dim}\left((S / \mathfrak{p})_{\mathfrak{q}}\right) .
$$

This is the same as

$$
\operatorname{dim}\left(M_{\mathfrak{q}}\right)=\operatorname{dim}\left(M_{\mathfrak{p}}\right)+\operatorname{dim}\left(\left(A_{0} / \mathfrak{p}\right)_{\mathfrak{q}}\right) .
$$

2.4. The special case of $A_{0}$ regular. Let $(R, \mathfrak{m})$ be a local Noetherian ring and $M$ an $R$-module. Then we define

$$
\operatorname{codepth}_{R}(M):=\operatorname{dim}_{R}(M)-\operatorname{depth}_{R}(M) .
$$

As usual the depth of the zero module is defined to be $\infty$, and the dimension of the zero module is $-\infty$, implying that the codepth of the zero module is $-\infty$.

The following proposition extends a result by Auslander [4, (6.11.2)] to the graded case. 
Proposition. Let $A$ and $M$ be as above and assume that $A_{0}$ is a homomorphic image of a regular ring. The function $\varphi: \operatorname{Spec}\left(A_{0}\right) \longrightarrow \mathbb{N}$ defined by

$$
\varphi(\mathfrak{p})=\operatorname{codepth}_{\left(A_{0}\right)_{\mathfrak{p}}}\left(M_{\mathfrak{p}}\right) \text { for all } \mathfrak{p} \in \operatorname{Spec}\left(A_{0}\right)
$$

is upper semicontinuous, that is, for all $n \in \mathbb{N}$, the set

$$
U_{C_{n}}^{0}(M)=\left\{\mathfrak{p} \in \operatorname{Spec}\left(A_{0}\right) \mid \operatorname{codepth}_{\left(A_{0}\right)_{\mathfrak{p}}}\left(M_{\mathfrak{p}}\right) \leq n\right\}
$$

is open in $\operatorname{Spec}\left(A_{0}\right)$.

Proof. If $A_{0}$ is a homomorphic image of a regular ring $R_{0}$, then the dimension and the depth of the $R_{0}$-module $M$ are identical to the dimension and depth of $M$ considered as an $R_{0}$-module. If we show that the set

$$
\widetilde{U}_{C_{n}}^{0}(M)=\left\{\mathfrak{q} \in \operatorname{Spec}\left(R_{0}\right) \mid \operatorname{codepth}_{\left(R_{0}\right)_{\mathfrak{q}}}\left(M_{\mathfrak{q}}\right) \leq n\right\}
$$

is open in $\operatorname{Spec}\left(R_{0}\right)$ (where $M$ is considered an $R_{0}$-module), then the corresponding set for the $A_{0}$-module $M$ is given by

$$
U_{C_{n}}^{0}(M)=\widetilde{U}_{C_{n}}^{0}(M) \cap V(J),
$$

where $A_{0}=R_{0} / J$. Thus we may assume that $A_{0}$ is a regular ring. We may also assume that $A$ is a polynomial ring over $A_{0}$ equipped with the standard grading.

Let $\mathfrak{p} \in \operatorname{Spec}\left(A_{0}\right)$. By Proposition 1.2.3, the Auslander-Buchsbaum formula holds:

$$
\operatorname{depth}_{\left(A_{0}\right)_{\mathfrak{p}}}\left(M_{\mathfrak{p}}\right)=\operatorname{depth}\left(\left(A_{0}\right)_{\mathfrak{p}}\right)-\operatorname{projdim}_{\left(A_{0}\right)_{\mathfrak{p}}}\left(M_{\mathfrak{p}}\right) .
$$

Let $I=\operatorname{ann}_{A_{0}}(M)$. By Lemma 1.1.3, $I_{\mathfrak{p}}=\operatorname{ann}_{\left(A_{0}\right)_{\mathfrak{p}}}\left(M_{\mathfrak{p}}\right)$, and we have that

$$
\operatorname{dim}_{\left(A_{0}\right)_{\mathfrak{p}}}\left(M_{\mathfrak{p}}\right)=\operatorname{dim}\left(\left(A_{0}\right)_{\mathfrak{p}}\right)-\operatorname{ht}\left(I\left(A_{0}\right)_{\mathfrak{p}}\right) .
$$

Suppose that $\mathfrak{p} \in \operatorname{Spec}\left(A_{0}\right)$ is such that

$$
\operatorname{codepth}_{\left(A_{0}\right)_{\mathfrak{p}}}\left(M_{\mathfrak{p}}\right) \leq n .
$$

If $M_{\mathfrak{p}}=0$, then $\mathfrak{p} \nsupseteq I$. Take an element $a \in I \cap\left(A_{0} \backslash \mathfrak{p}\right)$. Then for all

$$
\mathfrak{q} \in D_{a}=\left\{\mathfrak{w} \in \operatorname{Spec}\left(A_{0}\right) \mid a \notin \mathfrak{w}\right\}
$$

we have that $M_{\mathfrak{q}}=0$ and $\operatorname{codepth}_{\left(A_{0}\right)_{\mathfrak{q}}}\left(M_{\mathfrak{q}}\right)=-\infty \leq n$.

If $M_{\mathfrak{p}} \neq 0$ pick an element $a_{1} \in A_{0} \backslash \mathfrak{p}$ so that $\left(A_{0}\right)_{\mathfrak{p}}$ and $\left(A_{0}\right)_{a_{1}}$ have the same minimal primes and put $U_{1}=D_{a_{1}}=\left\{\mathfrak{w} \in \operatorname{Spec}\left(A_{0}\right) \mid a_{1} \notin \mathfrak{w}\right\}$. Then for all $\mathfrak{q} \in U_{1} \cap V^{0}(I)$,

$$
\operatorname{ht}\left(I\left(A_{0}\right)_{\mathfrak{q}}\right) \geq \operatorname{ht}\left(I\left(A_{0}\right)_{\mathfrak{p}}\right) .
$$

Let $\operatorname{projdim}_{\left(A_{0}\right)_{\mathfrak{p}}}\left(M_{\mathfrak{p}}\right)=t$. Then by Proposition 2.2 there is an open subset $U_{2}$ in $\operatorname{Spec}\left(A_{0}\right)$ so that

$$
\operatorname{projdim}_{\left(A_{0}\right)_{\mathfrak{q}}}\left(M_{\mathfrak{q}}\right) \leq t \text { for all } \mathfrak{q} \in U_{2} .
$$

Using the Auslander-Buchsbaum formula and the fact that $A_{0}$ is regular, we obtain for all $\mathfrak{q} \in U_{2} \cap U_{1} \cap V^{0}(I)$ :

$$
\begin{aligned}
\operatorname{codepth}_{\left(A_{0}\right)_{\mathfrak{q}}}\left(M_{\mathfrak{q}}\right) & =\operatorname{dim}_{\left(A_{0}\right)_{\mathfrak{q}}}\left(M_{\mathfrak{q}}\right)-\operatorname{depth}_{\left(A_{0}\right)_{\mathfrak{q}}}\left(M_{\mathfrak{q}}\right) \\
& =\operatorname{dim}\left(\left(A_{0}\right)_{\mathfrak{q}}\right)-\operatorname{ht}\left(I\left(A_{0}\right)_{\mathfrak{q}}\right)-\operatorname{dim}\left(\left(A_{0}\right)_{\mathfrak{q}}\right)+\operatorname{projdim}_{\left(A_{0}\right)_{\mathfrak{q}}}\left(M_{\mathfrak{q}}\right) \\
& =\operatorname{projdim}\left(A_{0}\right)_{\mathfrak{q}}\left(M_{\mathfrak{q}}\right)-\operatorname{ht}\left(I\left(A_{0}\right)_{\mathfrak{q}}\right) .
\end{aligned}
$$


This implies that for all $\mathfrak{q} \in U=U_{1} \cap U_{2}$,

$$
\operatorname{codepth}_{\left(A_{0}\right)_{\mathfrak{q}}}\left(M_{\mathfrak{q}}\right) \leq \operatorname{codepth}_{\left(A_{0}\right)_{\mathfrak{p}}}\left(M_{\mathfrak{p}}\right)
$$

and it follows that $U_{C_{n}}^{0}(M)$ is an open subset of $\operatorname{Spec}\left(A_{0}\right)$.

2.5. A local formula. Using the fact that a complete local Noetherian ring is the homomorphic image of a regular local ring, we obtain a result similar to 4 , (6.11.5)]:

Lemma. Let $A$ be a Noetherian graded homogeneous ring and let $M$ be a finitely generated graded $A$-module. Then for all prime ideals $\mathfrak{p}, \mathfrak{q} \in \operatorname{Spec}\left(A_{0}\right)$ with $\mathfrak{p} \subseteq \mathfrak{q}$ we have that

$$
\operatorname{codepth}_{\left(A_{0}\right)_{\mathfrak{q}}}\left(M_{\mathfrak{q}}\right) \geq \operatorname{codepth}_{\left(A_{0}\right)_{\mathfrak{p}}}\left(M_{\mathfrak{p}}\right) .
$$

Proof. By replacing $A_{0}$ by $\left(A_{0}\right)_{\mathfrak{q}}$ (and $A$ by $\left.A_{\mathfrak{q}}\right)$ we may assume that $\left(A_{0}, \mathfrak{m}_{0}\right)$ is a local ring. Then we have to show

$$
\operatorname{codepth}_{A_{0}}(M) \geq \operatorname{codepth}_{\left(A_{0}\right)_{\mathfrak{p}}}\left(M_{\mathfrak{p}}\right) .
$$

Let $\widehat{\mathfrak{p}} \in \operatorname{Spec}\left(\widehat{A}_{0}\right)$ be a minimal prime ideal over $\mathfrak{p} \widehat{A}_{0}$. Then $\widehat{\mathfrak{p}} \cap A_{0}=\mathfrak{p}$ and $\left(\widehat{A}_{0}\right)_{\widehat{p}}$ is flat over $\left(A_{0}\right)_{\mathfrak{p}}$ with trivial special fiber. Moreover,

$$
\begin{aligned}
M_{\mathfrak{p}} \otimes_{\left(A_{0}\right)_{\mathfrak{p}}}\left(\widehat{A}_{0}\right)_{\widehat{\mathfrak{p}}} & =\left(\bigoplus_{i \in \mathbb{Z}}\left(M_{i}\right)_{\mathfrak{p}}\right) \otimes_{\left(A_{0}\right)_{\mathfrak{p}}}\left(\widehat{A}_{0}\right)_{\hat{\mathfrak{p}}} \\
& =\bigoplus_{i \in \mathbb{Z}}\left(\left(M_{i}\right)_{\mathfrak{p}} \otimes_{\left(A_{0}\right)_{\mathfrak{p}}}\left(\widehat{A}_{0}\right)_{\hat{\mathfrak{p}}}\right) \\
& \cong \bigoplus_{i \in \mathbb{Z}}\left(\widehat{M}_{i}\right)_{\widehat{\mathfrak{p}}},
\end{aligned}
$$

where $\widehat{M}_{i} \cong M_{i} \otimes_{A_{0}} \widehat{A}_{0}$. We have that

$$
\begin{gathered}
\operatorname{depth}_{A_{0}}(M)=\inf \left\{\operatorname{depth}_{A_{0}}\left(M_{i}\right) \mid M_{i} \neq 0\right\}, \\
\operatorname{dim}_{A_{0}}(M)=\sup \left\{\operatorname{dim}_{A_{0}}\left(M_{i}\right) \mid i \in \mathbb{Z}\right\} .
\end{gathered}
$$

By [8, Theorem 23.3], for all $i \in \mathbb{Z}$,

$$
\begin{aligned}
\operatorname{depth}_{\left(\widehat{A}_{0}\right)_{\hat{\mathfrak{p}}}}\left(\left(\widehat{M}_{i}\right)_{\widehat{\mathfrak{p}}}\right) & =\operatorname{depth}_{\left(A_{0}\right)_{\mathfrak{p}}}\left(\left(M_{i}\right)_{\mathfrak{p}}\right)+\operatorname{depth}\left(\left(\widehat{A}_{0}\right)_{\widehat{\mathfrak{p}}} / \mathfrak{p}\left(\widehat{A}_{0}\right)_{\widehat{\mathfrak{p}}}\right) \\
& =\operatorname{depth}_{\left(A_{0}\right)_{\mathfrak{p}}}\left(\left(M_{i}\right)_{\mathfrak{p}}\right),
\end{aligned}
$$

and by [8, Theorem 15.1],

$$
\begin{aligned}
\operatorname{dim}_{\left(\widehat{A}_{0}\right)_{\hat{\mathfrak{p}}}}\left(\left(\widehat{M}_{i}\right)_{\widehat{\mathfrak{p}}}\right) & =\operatorname{dim}_{\left(A_{0}\right)_{\mathfrak{p}}}\left(\left(M_{i}\right)_{\mathfrak{p}}\right)+\operatorname{dim}\left(\left(\widehat{A}_{0}\right)_{\widehat{\mathfrak{p}}} / \mathfrak{p}\left(\widehat{A}_{0}\right)_{\widehat{\mathfrak{p}}}\right) \\
& =\operatorname{dim}_{\left(A_{0}\right)_{\mathfrak{p}}}\left(\left(M_{i}\right)_{\mathfrak{p}}\right) .
\end{aligned}
$$

Let

$$
\widetilde{M}:=\bigoplus_{i \in \mathbb{Z}} \widehat{M}_{i} \cong M \otimes_{A_{0}} \widehat{A}_{0}
$$

and note that $\widetilde{M}$ is a finitely generated graded module over the Noetherian homogeneous graded ring

$$
\widetilde{A}:=A \otimes_{A_{0}} \widehat{A}_{0} .
$$

The computation above shows that

$$
\operatorname{codepth}_{\left(\widehat{A}_{0}\right)_{\hat{\mathfrak{p}}}}\left(\widetilde{M}_{\widehat{\mathfrak{p}}}\right)=\operatorname{codepth}_{\left(A_{0}\right)_{\mathfrak{p}}}\left(M_{\mathfrak{p}}\right)=: n .
$$

Since $\widehat{A}_{0}$ is a homomorphic image of a regular local ring, by Proposition 2.3 the set $U_{C_{n-1}}^{0}(\widetilde{M})$ is open in $\operatorname{Spec}\left(\widehat{A}_{0}\right)$. This implies that

$$
\operatorname{codepth}_{\widehat{A}_{0}}(\tilde{M}) \geq \operatorname{codepth}_{\left(\widehat{A}_{0}\right)_{\hat{\mathfrak{p}}}}\left(\widetilde{M}_{\widehat{\mathfrak{p}}}\right) \text {. }
$$


The same argument as above shows that

$$
\operatorname{codepth}_{\widehat{A}_{0}}(\widetilde{M})=\operatorname{codepth}_{A_{0}}(M)
$$

which proves the claim

$$
\operatorname{codepth}_{A_{0}}(M) \geq \operatorname{codepth}_{\left(A_{0}\right)_{\mathfrak{p}}}\left(M_{\mathfrak{p}}\right) .
$$

2.6. Formulas for depth and codepth. In this section we make the same assumption as at the beginning, namely, $A$ is a positively graded Noetherian homogeneous ring and $M$ is a finitely generated graded $A$-module. The following proposition is the graded version of [4, (6.10.6)]:

2.6.1. Proposition. Let $A$ and $M$ be as above and assume that $A$ is excellent. Then for every $\mathfrak{p} \in \operatorname{Spec}\left(A_{0}\right)$ there is an open subset $U^{0} \subseteq \operatorname{Spec}\left(A_{0}\right)$ with $\mathfrak{p} \in U^{0}$ so that for all $\mathfrak{q} \in U^{0} \cap V^{0}(\mathfrak{p})$,

$$
\operatorname{depth}_{\left(A_{0}\right)_{\mathfrak{q}}}\left(M_{\mathfrak{q}}\right)=\operatorname{depth}_{\left(A_{0}\right)_{\mathfrak{p}}}\left(M_{\mathfrak{p}}\right)+\operatorname{depth}\left(\left(A_{0}\right)_{\mathfrak{q}} / \mathfrak{p}\left(A_{0}\right)_{\mathfrak{q}}\right) .
$$

Proof. Let $\mathfrak{p} \in \operatorname{Spec}\left(A_{0}\right)$. Then by Lemma 2.5 for all $\mathfrak{q} \in V^{0}(\mathfrak{p})$,

$$
\operatorname{codepth}_{\left(A_{0}\right)_{\mathfrak{q}}}\left(M_{\mathfrak{q}}\right) \geq \operatorname{codepth}_{\left(A_{0}\right)_{\mathfrak{p}}}\left(M_{\mathfrak{p}}\right),
$$

or equivalently,

(*) $\quad \operatorname{dim}_{\left(A_{0}\right)_{\mathfrak{q}}}\left(M_{\mathfrak{q}}\right)-\operatorname{depth}_{\left(A_{0}\right)_{\mathfrak{q}}}\left(M_{\mathfrak{q}}\right) \geq \operatorname{dim}_{\left(A_{0}\right)_{\mathfrak{p}}}\left(M_{\mathfrak{p}}\right)-\operatorname{depth}_{\left(A_{0}\right)_{\mathfrak{p}}}\left(M_{\mathfrak{p}}\right)$.

According to Proposition 2.3 there is an open subset $U_{1} \subseteq \operatorname{Spec}\left(A_{0}\right)$ with $\mathfrak{p} \in U_{1}$ so that for all $\mathfrak{q} \in U_{1} \cap V^{0}(\mathfrak{p})$,

$$
\operatorname{dim}_{\left(A_{0}\right)_{\mathfrak{q}}}\left(M_{\mathfrak{q}}\right)=\operatorname{dim}_{\left(A_{0}\right)_{\mathfrak{p}}}\left(M_{\mathfrak{p}}\right)+\operatorname{dim}\left(\left(A_{0} / \mathfrak{p}\right)_{\mathfrak{q}}\right) .
$$

Since $A_{0}$ is excellent, there is an open subset $U_{2} \subseteq \operatorname{Spec}\left(A_{0}\right)$ so that $\mathfrak{p} \in U_{2}$, and for all $\mathfrak{q} \in U_{2} \cap V^{0}(\mathfrak{p})$ the local ring

$$
\left(A_{0} / \mathfrak{p}\right)_{\mathfrak{q}} \text { is Cohen-Macaulay. }
$$

There is also an open subset $U_{3} \subseteq \operatorname{Spec}\left(A_{0}\right)$ so that $\mathfrak{p} \in U_{3}$, and for all $\mathfrak{q} \in$ $U_{3} \cap V^{0}(\mathfrak{p})$ we have equality on the set of minimal primes:

$$
\operatorname{Min}_{\left(A_{0}\right)_{\mathfrak{q}}}\left(I\left(A_{0}\right)_{\mathfrak{q}}\right)=\operatorname{Min}_{\left(A_{0}\right)_{\mathfrak{p}}}\left(I\left(A_{0}\right)_{\mathfrak{p}}\right)
$$

where $I:=\operatorname{ann}_{A_{0}}(M)$ denotes the $A_{0}$-annihilator of $M$. In particular, for all $\mathfrak{q} \in U_{3} \cap V^{0}(\mathfrak{p})$,

$$
\operatorname{ht}\left(I\left(A_{0}\right)_{\mathfrak{q}}\right)=\operatorname{ht}\left(I\left(A_{0}\right)_{\mathfrak{p}}\right) .
$$

Put $\widetilde{U}_{1}=U_{1} \cap U_{2} \cap U_{3}$; then for all $\mathfrak{q} \in \widetilde{U}_{1} \cap V^{0}(\mathfrak{p})$,

$$
\operatorname{dim}_{\left(A_{0}\right)_{\mathfrak{q}}}\left(M_{\mathfrak{q}}\right)=\operatorname{dim}\left(\left(A_{0} / I\right)_{\mathfrak{q}}\right) \quad \text { and } \quad \operatorname{dim}_{\left(A_{0}\right)_{\mathfrak{p}}}\left(M_{\mathfrak{p}}\right)=\operatorname{dim}\left(\left(A_{0} / I\right)_{\mathfrak{p}}\right) .
$$

Since $A$ is excellent, the ring $A_{0}$ is universally catenary, and for all $\mathfrak{q} \in \widetilde{U}_{1} \cap V^{0}(\mathfrak{p})$,

$$
\operatorname{dim}\left(\left(A_{0} / I\right)_{\mathfrak{q}}\right)-\operatorname{dim}\left(\left(A_{0} / I\right)_{\mathfrak{p}}\right)=\operatorname{dim}\left(\left(A_{0} / \mathfrak{p}\right)_{\mathfrak{q}}\right)=\operatorname{depth}\left(\left(A_{0} / \mathfrak{p}\right)_{\mathfrak{q}}\right) .
$$

From $(*)$ we obtain

$$
\operatorname{depth}_{\left(A_{0}\right)_{\mathfrak{q}}}\left(M_{\mathfrak{q}}\right)-\operatorname{depth}_{\left(A_{0}\right)_{\mathfrak{p}}}\left(M_{\mathfrak{p}}\right) \leq \operatorname{depth}\left(\left(A_{0} / \mathfrak{p}\right)_{\mathfrak{q}}\right)
$$

for all $\mathfrak{q} \in \widetilde{U}_{1} \cap V^{0}(\mathfrak{p})$.

In order to prove the other inequality,

$$
\operatorname{depth}_{\left(A_{0}\right)_{\mathfrak{q}}}\left(M_{\mathfrak{q}}\right)-\operatorname{depth}_{\left(A_{0}\right)_{\mathfrak{p}}}\left(M_{\mathfrak{p}}\right) \geq \operatorname{depth}\left(\left(A_{0} / \mathfrak{p}\right)_{\mathfrak{q}}\right)
$$


assume that $\operatorname{depth}_{\left(A_{0}\right)_{\mathfrak{p}}}\left(M_{\mathfrak{p}}\right)=t$ and let $f_{1}, \ldots, f_{t} \in \mathfrak{p}$ be such that $f_{1}, \ldots, f_{t}$ is a regular sequence on $M_{\mathfrak{p}}$. A prime avoidance argument shows that there is an element $a \in A_{0} \backslash \mathfrak{p}$ so that $f_{1}, \ldots, f_{t}$ is a regular sequence on $M_{a}$. (The argument again makes use of the fact that the sets $\operatorname{Ass}_{A_{0}}(M)$ and $\operatorname{Ass}_{A_{0}}\left(M /\left(f_{1}, \ldots, f_{i}\right) M\right)$ for all $1 \leq i \leq t$ are finite.)

Put

$$
\bar{M}:=M /\left(f_{1}, \ldots, f_{t}\right) M,
$$

and consider the associated graded module

$$
\operatorname{gr}_{\mathfrak{p}}(\bar{M})=\bigoplus_{i \in \mathbb{N}} \mathfrak{p}^{i} \bar{M} / \mathfrak{p}^{i+1} \bar{M} .
$$

The module $\bar{M}$ is finitely generated over $A$, and $\operatorname{gr}_{\mathfrak{p}}(\bar{M})$ is a finitely generated $\operatorname{gr}_{\mathfrak{p}}(A)$-module. Also note that $\operatorname{gr}_{\mathfrak{p}}(A)$ is a finitely generated algebra over $A / \mathfrak{p} A$ and that $A / \mathfrak{p} A$ is a finitely generated algebra over $A_{0} / \mathfrak{p}$. Thus $\operatorname{gr}_{\mathfrak{p}}(A)$ is a finitely generated $A_{0} / \mathfrak{p}$-algebra. By [8, Theorem 24.1] there is an element $b \in A_{0} \backslash \mathfrak{p}$ so that the $\left(A_{0} / \mathfrak{p}\right)_{b}$-module

$$
\operatorname{gr}_{\mathfrak{p}}(\bar{M})_{b}=\bigoplus_{i \in \mathbb{N}}\left(\mathfrak{p}^{i} \bar{M} / \mathfrak{p}^{i+1} \bar{M}\right)_{b}
$$

is free. Set $\widetilde{U}_{2}=D_{b}=\left\{\mathfrak{q} \in \operatorname{Spec}\left(A_{0}\right) \mid b \notin \mathfrak{q}\right\}$ and fix a prime ideal $\mathfrak{q} \in \widetilde{U}_{2} \cap V^{0}(\mathfrak{p})$. Assume that

$$
\operatorname{depth}\left(\left(A_{0} / \mathfrak{p}\right)\right)_{\mathfrak{q}}=s,
$$

and let $g_{1}, \ldots, g_{s} \in \mathfrak{q}$ be such that $g_{1}, \ldots, g_{s}$ is a regular sequence on $\left(A_{0} / \mathfrak{p}\right)_{\mathfrak{q}}$.

Claim 1. $g_{1}$ is a regular element on $\bar{M}_{\mathfrak{q}}$.

Claim 2. Set $N_{1}:=\bar{M}_{\mathfrak{q}} / g_{1} \bar{M}_{\mathfrak{q}}$; then $\operatorname{gr}_{\mathfrak{p}}\left(N_{1}\right) \cong \operatorname{gr}_{\mathfrak{p}}\left(\bar{M}_{\mathfrak{q}}\right) / g_{1} \operatorname{gr}_{\mathfrak{p}}\left(\bar{M}_{\mathfrak{q}}\right)$.

Assuming the claims, we finish the proof. From the second claim it follows that $\operatorname{gr}_{\mathfrak{p}}\left(N_{1}\right)$ is a free $\left(A_{0} /\left(g_{1}, \mathfrak{p}\right) A_{0}\right)_{\mathfrak{q}}$-module. Since $g_{2}$ is a regular element on $\left(A_{0} /\left(g_{1}, \mathfrak{p}\right) A_{0}\right)_{\mathfrak{q}}$, we may apply Claims 1 and 2 to $N_{1}$. Note that $N_{1}$ is also a finitely generated graded $A_{\mathfrak{q}}$-module. This yields that $g_{2}$ is a regular element on $N_{1}$ and that with $N_{2}=N_{1} / g_{2} N_{1}$,

$$
\operatorname{gr}_{\mathfrak{p}}\left(N_{2}\right) \cong \operatorname{gr}_{\mathfrak{p}}\left(N_{1}\right) / g_{2} \operatorname{gr}_{\mathfrak{p}}\left(N_{1}\right) \text {. }
$$

An induction argument yields that $g_{1}, \ldots, g_{s}$ is a regular sequence on $\bar{M}_{\mathfrak{q}}$, and we have that

$$
\operatorname{depth}_{\left(A_{0}\right)_{\mathfrak{q}}}\left(M_{\mathfrak{q}}\right) \geq \operatorname{depth}_{\left(A_{0}\right)_{\mathfrak{p}}}\left(M_{\mathfrak{p}}\right)+\operatorname{depth}\left(\left(A_{0} / \mathfrak{p}\right)_{\mathfrak{q}} .\right.
$$

This inequality holds for all $\mathfrak{q} \in \widetilde{U}_{2} \cap V^{0}(\mathfrak{p})$. Assuming the claims the proposition is now proved with $U^{0}=\widetilde{U}_{1} \cap \widetilde{U}_{2}$.

In order to prove the claims, set $g=g_{1}$ and $N=N_{1}$.

Proof of Claim 1. Let $z \in \bar{M}_{\mathfrak{q}}$ with $g z=0$. Consider the image $\bar{z}$ of $z$ in $\bar{M}_{\mathfrak{q}} / \mathfrak{p} \bar{M}_{\mathfrak{q}}$. Since $\bar{M}_{\mathfrak{q}} / \mathfrak{p} \bar{M}_{\mathfrak{q}}$ is a free module over $\left(A_{0} / \mathfrak{p}\right)_{\mathfrak{q}}$ and since $g$ is regular on $\left(A_{0} / \mathfrak{p}\right)_{\mathfrak{q}}$, we obtain that $\bar{z}=0$ and $z \in \mathfrak{p} \bar{M}_{\mathfrak{q}}$. Now consider the image of $z$ in $\mathfrak{p} \bar{M}_{\mathfrak{q}} / \mathfrak{p}^{2} \bar{M}_{\mathfrak{q}}$ and repeat the argument. This yields

$$
z \in \bigcap_{j=0}^{\infty} \mathfrak{p}^{j} \bar{M}_{\mathfrak{q}} .
$$


Note that

$$
\bar{M}_{\mathfrak{q}}=\bigoplus_{i \in \mathbb{Z}}\left(\bar{M}_{i}\right)_{\mathfrak{q}} \quad \text { with } \quad\left(\bar{M}_{i}\right)_{\mathfrak{q}}=\left(M_{i}\right)_{\mathfrak{q}} /\left(f_{1}, \ldots, f_{t}\right)\left(M_{i}\right)_{\mathfrak{q}}
$$

In particular,

$$
\mathfrak{p}^{j} \bar{M}_{\mathfrak{q}}=\bigoplus_{i \in \mathbb{Z}} \mathfrak{p}^{j}\left(\bar{M}_{i}\right)_{\mathfrak{q}}
$$

and every $\left(\bar{M}_{i}\right)_{\mathfrak{q}}$ is a finitely generated $\left(A_{0}\right)_{\mathfrak{q}}$-module. This shows that $z=0$.

Proof of Claim 2. By assumption, we have that $\operatorname{gr}_{\mathfrak{p}}\left(\bar{M}_{\mathfrak{q}}\right)$ is a free $\left(A_{0} / \mathfrak{p}\right)_{\mathfrak{q}}$ - module and $\mathfrak{p}^{j} \bar{M}_{\mathfrak{q}} / \mathfrak{p}^{j+1} \bar{M}_{\mathfrak{q}}$ is a direct summand of $\operatorname{gr}_{\mathfrak{p}}\left(\bar{M}_{\mathfrak{q}}\right)$. Thus $\mathfrak{p}^{j} \bar{M}_{\mathfrak{q}} / \mathfrak{p}^{j+1} \bar{M}_{\mathfrak{q}}$ is a free $\left(A_{0} / \mathfrak{p}\right)_{\mathfrak{q}}$-module and $g$ is regular on $\left(A_{0} / \mathfrak{p}\right)_{\mathfrak{q}}$. Therefore

$$
\mathfrak{p}^{j} \bar{M}_{\mathfrak{q}} \cap g \bar{M}_{\mathfrak{q}}=g \mathfrak{p}^{j} \bar{M}_{\mathfrak{q}}
$$

and thus

$$
\begin{aligned}
\mathfrak{p}^{j} \bar{M}_{\mathfrak{q}} / g \mathfrak{p}^{j} \bar{M}_{\mathfrak{q}} & \cong \mathfrak{p}^{j} \bar{M}_{\mathfrak{q}} /\left(\mathfrak{p}^{j} \bar{M}_{\mathfrak{q}} \cap g \bar{M}_{\mathfrak{q}}\right) \\
& \cong \mathfrak{p}^{j}\left(\bar{M}_{\mathfrak{q}} / g \bar{M}_{\mathfrak{q}}\right) .
\end{aligned}
$$

From the commutative diagram



we obtain that

$$
\begin{aligned}
\operatorname{gr}_{\mathfrak{p}}(N) & =\bigoplus_{j \in \mathbb{N}} \mathfrak{p}^{j} N / \mathfrak{p}^{j+1} N \\
& \cong \bigoplus_{j \in \mathbb{N}} \mathfrak{p}^{j} \bar{M}_{\mathfrak{q}} /\left(g \mathfrak{p}^{j} \bar{M}_{\mathfrak{q}}+\mathfrak{p}^{j+1} \bar{M}_{\mathfrak{q}}\right) \\
& \cong \bigoplus_{j \in \mathbb{N}}\left(\mathfrak{p}^{j} \bar{M}_{\mathfrak{q}} / \mathfrak{p}^{j+1} \bar{M}_{\mathfrak{q}}\right) / g\left(\mathfrak{p}^{j} \bar{M}_{\mathfrak{q}} / \mathfrak{p}^{j+1} \bar{M}_{\mathfrak{q}}\right) \\
& \cong \operatorname{gr}_{\mathfrak{p}}\left(\bar{M}_{\mathfrak{q}}\right) / g\left(\operatorname{gr}\left(\bar{M}_{\mathfrak{q}}\right) .\right.
\end{aligned}
$$

This proves the claim, and finishes the proof.

Similar to [4, (6.11.8.1)] we have in the graded case:

2.6.2. Corollary. Let $A$ and $M$ be as above and assume that $A$ is excellent. Then for every $\mathfrak{p} \in \operatorname{Spec}\left(A_{0}\right)$ there is an open subset $U^{0} \subseteq \operatorname{Spec}\left(A_{0}\right)$ with $\mathfrak{p} \in U^{0}$, so that for all $\mathfrak{q} \in U^{0} \cap V^{0}(\mathfrak{p})$,

$$
\operatorname{codepth}_{\left(A_{0}\right)_{\mathfrak{q}}}\left(M_{\mathfrak{q}}\right)=\operatorname{codepth}_{\left(A_{0}\right)_{\mathfrak{p}}}\left(M_{\mathfrak{p}}\right)+\operatorname{codepth}\left(\left(A_{0}\right)_{\mathfrak{q}} / \mathfrak{p}\left(A_{0}\right)_{\mathfrak{q}}\right) .
$$

Proof. Let $\mathfrak{p} \in \operatorname{Spec}\left(A_{0}\right)$ and let $U_{1}^{0}$ be as in Proposition 2.6.1 so that $\mathfrak{p} \in U_{1}^{0}$, and for all $\mathfrak{q} \in U_{1}^{0} \cap V^{0}(\mathfrak{p})$,

$$
\operatorname{depth}_{\left(A_{0}\right)_{\mathfrak{q}}}\left(M_{\mathfrak{q}}\right)=\operatorname{depth}_{\left(A_{0}\right)_{\mathfrak{p}}}\left(M_{\mathfrak{p}}\right)+\operatorname{depth}\left(\left(A_{0}\right)_{\mathfrak{q}} / \mathfrak{p}\left(A_{0}\right)_{\mathfrak{q}}\right) .
$$

By Proposition 2.3 there is an open subset $U_{2}^{0}$ in $\operatorname{Spec}\left(A_{0}\right)$, so that $\mathfrak{p} \in U_{2}^{0}$, and for all $\mathfrak{q} \in U_{2}^{0} \cap V^{0}(\mathfrak{p})$,

$$
\operatorname{dim}_{\left(A_{0}\right)_{\mathfrak{q}}}\left(M_{\mathfrak{q}}\right)=\operatorname{dim}_{\left(A_{0}\right)_{\mathfrak{p}}}\left(M_{\mathfrak{p}}\right)+\operatorname{dim}\left(\left(A_{0} / \mathfrak{p}\right)_{\mathfrak{q}}\right) .
$$

Thus with $U^{0}=U_{1}^{0} \cap U_{2}^{0}$ we have that $\mathfrak{p} \in U^{0}$, and for all $\mathfrak{q} \in U^{0} \cap V^{0}(\mathfrak{p})$,

$$
\operatorname{codepth}_{\left(A_{0}\right)_{\mathfrak{q}}}\left(M_{\mathfrak{q}}\right)=\operatorname{codepth}_{\left(A_{0}\right)_{\mathfrak{p}}}\left(M_{\mathfrak{p}}\right)+\operatorname{codepth}\left(\left(A_{0}\right)_{\mathfrak{q}} / \mathfrak{p}\left(A_{0}\right)_{\mathfrak{q}}\right) .
$$


We are now ready to prove the graded version of [4, (6.11.2)(a)].

2.6.3. Theorem. Let $A=\bigoplus_{i \in \mathbb{N}} A_{i}$ be an excellent graded homogeneous ring and let $M=\bigoplus_{i \in \mathbb{Z}} M_{i}$ be a finitely generated graded $A$-module. Then for all $n \in \mathbb{N}$ the set

$$
U_{C_{n}}^{0}(M)=\left\{\mathfrak{p} \in \operatorname{Spec}\left(A_{0}\right) \mid \operatorname{codepth}_{\left(A_{0}\right)_{\mathfrak{p}}}\left(M_{\mathfrak{p}}\right) \leq n\right\}
$$

is open in $\operatorname{Spec}\left(A_{0}\right)$.

Proof. According to Nagata's criterion on openness of loci (see [8, Theorem 24.2]) we need to show:

(a) If $\mathfrak{p}, \mathfrak{q} \in \operatorname{Spec}\left(A_{0}\right)$ with $\mathfrak{q} \subseteq \mathfrak{p}$ and $\mathfrak{p} \in U_{C_{n}}^{0}(M)$, then $\mathfrak{q} \in U_{C_{n}}^{0}(M)$.

(b) If $\mathfrak{p} \in U_{C_{n}}^{0}(M)$, then $U_{C_{n}}^{0}(M)$ contains a nonempty open subset of $V(\mathfrak{p})$.

(a) Let $\mathfrak{p}, \mathfrak{q} \in \operatorname{Spec}\left(A_{0}\right)$ with $\mathfrak{q} \subseteq \mathfrak{p}$. By Lemma 2.5

$$
\operatorname{codepth}_{\left(A_{0}\right)_{\mathfrak{p}}}\left(M_{\mathfrak{p}}\right) \geq \operatorname{codepth}_{\left(A_{0}\right)_{\mathfrak{q}}}\left(M_{\mathfrak{q}}\right),
$$

and thus $\mathfrak{p} \in U_{C_{n}}^{0}(M)$ implies that $\mathfrak{q} \in U_{C_{n}}^{0}(M)$.

(b) Let $\mathfrak{p} \in U_{C_{n}}^{0}(M)$. By Corollary 2.6.2 there is an open subset $U_{1}^{0}$ in $\operatorname{Spec}\left(A_{0}\right)$, so that $\mathfrak{p} \in U_{1}^{0}$, and for all $\mathfrak{q} \in U_{1}^{0} \cap V^{0}(\mathfrak{p})$,

$$
\operatorname{codepth}_{\left(A_{0}\right)_{\mathfrak{q}}}\left(M_{\mathfrak{q}}\right)=\operatorname{codepth}_{\left(A_{0}\right)_{\mathfrak{p}}}\left(M_{\mathfrak{p}}\right)+\operatorname{codepth}\left(\left(A_{0}\right)_{\mathfrak{q}} / \mathfrak{p}\left(A_{0}\right)_{\mathfrak{q}}\right) \text {. }
$$

Since $A$ and $A_{0}$ are excellent, there is an open subset $U_{2}^{0}$ in $\operatorname{Spec}\left(A_{0}\right)$, so that $\mathfrak{p} \in U_{2}^{0}$, and for all $\mathfrak{q} \in U_{2}^{0} \cap V^{0}(\mathfrak{p})$, the ring $\left(A_{0} / \mathfrak{p}\right)_{\mathfrak{q}}$ is Cohen-Macaulay. Therefore with $U^{0}=U_{1}^{0} \cap U_{2}^{0}$ we have that $\mathfrak{p} \in U^{0}$, and for all $\mathfrak{q} \in U^{0} \cap V^{0}(\mathfrak{p})$,

$$
\operatorname{codepth}_{\left(A_{0}\right)_{\mathfrak{q}}}\left(M_{\mathfrak{q}}\right)=\operatorname{codepth}_{\left(A_{0}\right)_{\mathfrak{p}}}\left(M_{\mathfrak{p}}\right) .
$$

This implies that $U^{0} \cap V^{0}(\mathfrak{p}) \subseteq U_{C_{n}}^{0}(M)$, and the theorem is proved.

2.6.4. Corollary. Let $A$ and $M$ be as in Theorem 2.6.3. Then the Cohen-Macaulay locus of the $A_{0}$-module $M$,

$$
U_{C M}^{0}(M)=U_{C_{0}}^{0}(M)=\left\{\mathfrak{p} \in \operatorname{Spec}\left(A_{0}\right) \mid M_{\mathfrak{p}} \text { is a CM module over }\left(A_{0}\right)_{\mathfrak{p}}\right\},
$$

is open in $\operatorname{Spec}\left(A_{0}\right)$.

\section{Openness of the $\left(S_{n}\right)$-LOCUS}

Throughout this section we assume that $R=A_{0}$ is the base ring of a graded Noetherian homogeneous ring $A=\bigoplus_{i>0} A_{i}$ and $M$ is a finitely generated graded $A$ module. This includes the case of a finitely generated module $M$ over a Noetherian $\operatorname{ring} R$. For those modules we prove that the openness of the $C_{n}$-loci of $M$ implies the openness of the $\left(S_{k}\right)$-loci of $M$. The argument is due to Grothendieck [4, (5.7.2) and $(6.11 .2)(\mathrm{b})]$, but we include it here for the convenience of the reader. The proof also shows that the $\left(S_{k}\right)$-loci of $M$ only depend on the $C_{n}$-loci of $M$ and on the annihilator of $M$, so that two $R$-modules $M$ and $N$ with the same annihilators and $C_{n}$-loci have identical $\left(S_{k}\right)$-loci.

Let $M$ be an $R$-module and suppose that for all $n \in \mathbb{N}_{0}$, the set

$$
U_{C_{n}}(M)=\left\{\mathfrak{p} \in \operatorname{Spec}(R) \mid \operatorname{codepth}_{R_{\mathfrak{p}}}\left(M_{\mathfrak{p}}\right) \leq n\right\}
$$

is open in $\operatorname{Spec}(R)$. Define

$$
Z_{n}=V\left(\mathfrak{b}_{n}\right)=\operatorname{Spec}(R) \backslash U_{C_{n}}(M),
$$


where $\mathfrak{b}_{n} \subseteq R$ is a reduced ideal. Obviously, for all $n \in \mathbb{N}$,

$$
U_{C_{n}}(M) \subseteq U_{C_{n+1}}(M),
$$

and therefore

$$
Z_{n+1} \subseteq Z_{n} \quad \text { and } \quad \mathfrak{b}_{n} \subseteq \mathfrak{b}_{n+1}
$$

Since $R$ is Noetherian, there is an $m \in \mathbb{N}$ so that for all $t \in \mathbb{N}$,

$$
\mathfrak{b}_{m}=\mathfrak{b}_{m+t} \text { and } Z_{m}=Z_{m+t} .
$$

3.1. Lemma. Let $m \in \mathbb{N}$ be as above. Then $Z_{m}=\emptyset$.

Proof. If $\mathfrak{p} \in Z_{m}$, then $\mathfrak{p} \in Z_{m+t}$ for all $t \in \mathbb{N}$. By definition of $Z_{n+t}$,

$$
\operatorname{codepth}_{(R)_{\mathfrak{p}}}\left(M_{\mathfrak{p}}\right) \geq m+t \text { for all } t \in \mathbb{N} \text {. }
$$

But $\operatorname{codepth}_{R_{\mathfrak{p}}}\left(M_{\mathfrak{p}}\right) \leq \operatorname{dim}\left((R)_{\mathfrak{p}}\right) \leq \infty$, and therefore $Z_{m}=\emptyset$.

Recall that the $R$-module $M$ satisfies Serre's condition $\left(S_{k}\right)$ if for all $\mathfrak{p} \in \operatorname{Spec}(R)$,

$$
\operatorname{depth}_{R_{\mathfrak{p}}}\left(M_{\mathfrak{p}}\right) \geq \min \left(\operatorname{dim}\left(M_{\mathfrak{p}}\right), k\right) .
$$

From now on let $m$ denote the minimal $m \in \mathbb{N}$ with $Z_{m}=\emptyset$.

3.2. Lemma. With the assumptions as above put $\bar{R}=R / \operatorname{ann}_{R}(M)$ and let $k \in \mathbb{N}$. Then the $R$-module $M$ satisfies $\left(S_{k}\right)$ if and only if for all $0 \leq n<m$,

$$
\operatorname{ht}\left(\mathfrak{b}_{n} \bar{R}\right)>n+k .
$$

Proof. Suppose that $M$ satisfies $\left(S_{k}\right)$, and fix an integer $n$ with $0 \leq n<m$. Let $\mathfrak{p} \in \operatorname{Spec}(R)$ with $\mathfrak{b}_{n} \subseteq \mathfrak{p}$. Then $\mathfrak{p} \in Z_{n}$, and therefore

$$
\operatorname{codepth}_{R_{\mathfrak{p}}}\left(M_{\mathfrak{p}}\right)>n,
$$

or equivalently,

$$
\operatorname{dim}_{R_{\mathfrak{p}}}\left(M_{\mathfrak{p}}\right)-\operatorname{depth}_{R_{\mathfrak{p}}}\left(M_{\mathfrak{p}}\right)>n .
$$

Since $M$ satisfies $\left(S_{k}\right)$, we obtain that whenever

$$
\operatorname{dim}_{R_{\mathfrak{p}}}\left(M_{\mathfrak{p}}\right)-\operatorname{depth}_{R_{\mathfrak{p}}}\left(M_{\mathfrak{p}}\right) \neq 0,
$$

then

Thus, if $\mathfrak{p} \in Z_{n}$, then

$$
\operatorname{depth}_{R_{\mathfrak{p}}}\left(M_{\mathfrak{p}}\right) \geq k
$$

which implies that $\operatorname{ht}\left(\mathfrak{b}_{n} \bar{R}\right) \geq n+k$.

$$
\operatorname{dim}_{R_{\mathfrak{p}}}\left(M_{\mathfrak{p}}\right) \geq n+k,
$$

Conversely, fix an integer $k$ and assume that for all $0 \leq n<m$,

$$
\operatorname{ht}\left(\mathfrak{b}_{n} \bar{R}\right)>n+k \text {. }
$$

Let $\mathfrak{p} \in \operatorname{Spec}(R)$.

If $M_{\mathfrak{p}}=0$, then $\operatorname{depth}_{R_{\mathfrak{p}}}\left(M_{\mathfrak{p}}\right)=\infty$, and condition $(*)$ is satisfied.

Now assume $M_{\mathfrak{p}} \neq 0$. If $M_{\mathfrak{p}}$ is a Cohen-Macaulay $R$-module, then condition (*) is satisfied. Now assume that

$$
\operatorname{codepth}_{R_{\mathfrak{p}}}\left(M_{\mathfrak{p}}\right)>0,
$$

and let $n \in \mathbb{N}_{0}$ with

$$
\operatorname{codepth}_{R_{\mathfrak{p}}}\left(M_{\mathfrak{p}}\right)=n+1 \text {. }
$$

Thus $\mathfrak{p} \in Z_{n}$ and $\mathfrak{b}_{n} \subseteq \mathfrak{p}$. By assumption,

$$
\operatorname{ht}\left(\mathfrak{b}_{n} \bar{R}\right)>n+k \Rightarrow \operatorname{ht}\left(\mathfrak{b}_{n} \bar{R}_{\mathfrak{p}}\right)>n+k \Rightarrow \operatorname{dim}\left(\bar{R}_{\mathfrak{p}}\right)>n+k .
$$


This implies that

$$
\begin{aligned}
\operatorname{codepth}_{R_{\mathfrak{p}}}\left(M_{\mathfrak{p}}\right) & =n+1 \\
& =\operatorname{dim}\left(\bar{R}_{\mathfrak{p}}\right)-\operatorname{depth}_{R_{\mathfrak{p}}}\left(M_{\mathfrak{p}}\right) \\
& \geq n+1+k-\operatorname{depth}_{R_{\mathfrak{p}}}\left(M_{\mathfrak{p}}\right)
\end{aligned}
$$

and therefore

$$
\operatorname{depth}_{R_{\mathfrak{p}}}\left(M_{\mathfrak{p}}\right) \geq k .
$$

Thus $M_{\mathfrak{p}}$ satisfies condition $(*)$, and the $R$-module $M$ satisfies Serre's condition $\left(S_{k}\right)$.

For all $0 \leq n<m$ consider the closed subset of $\operatorname{Spec}(R)$,

$$
Y_{n, k}=\left\{\mathfrak{q} \in V\left(\mathfrak{b}_{n}\right) \mid \operatorname{ht}\left(\mathfrak{b}_{n} \bar{R}_{\mathfrak{q}}\right) \leq n+k\right\},
$$

and its complement

$$
V_{n, k}=\operatorname{Spec}(R)-Y_{n, k}
$$

an open subset of $\operatorname{Spec}(R)$. By Lemma 3.2

$$
U_{S_{k}}(M)=\bigcap_{0 \leq n<m} V_{n, k}
$$

is an open subset of $\operatorname{Spec}(R)$. We have shown:

3.3. Theorem. Let $M$ be an $R$-module as above. If for all $n \in \mathbb{N}_{0}$ the $C_{n}$-locus $U_{C_{n}}(M)$ is open in $\operatorname{Spec}(R)$, then for all $k \in \mathbb{N}$, the $\left(S_{k}\right)$-locus

$$
U_{S_{k}}(M)=\left\{\mathfrak{p} \in \operatorname{Spec}(R) \mid M_{\mathfrak{p}} \text { satisfies }\left(S_{k}\right)\right\}
$$

is open in $\operatorname{Spec}(R)$.

In the graded case the theorem states:

3.4. Corollary. Let $A=\bigoplus_{i \in \mathbb{N}} A_{i}$ be an excellent graded homogeneous ring and let $M=\bigoplus_{i \in \mathbb{Z}} M_{i}$ be a finitely generated graded $A$-module. Then for all $k \in \mathbb{N}$, the set

$$
U_{S_{k}}^{0}(M)=\left\{\mathfrak{p} \in \operatorname{Spec}\left(A_{0}\right) \mid \text { the }\left(A_{0}\right)_{\mathfrak{p}} \text {-module } M_{\mathfrak{p}} \text { satisfies }\left(S_{k}\right)\right\}
$$

is open in $\operatorname{Spec}\left(A_{0}\right)$.

The proof of the theorem also yields the following corollary:

3.5. Corollary. Suppose that $M$ and $N$ are $R$-modules as above. Assume that $\operatorname{ann}_{R}(M)=\operatorname{ann}_{R}(N)$ and that for all $n \in \mathbb{N}_{0}$, the sets $U_{C_{n}}(M)=U_{C_{n}}(N)$ are open in $\operatorname{Spec}(R)$. Then for all $k \in \mathbb{N}$,

$$
U_{S_{k}}(M)=U_{S_{k}}(N),
$$

and the $\left(S_{k}\right)$-loci are open subsets of $\operatorname{Spec}(R)$. 


\section{Stability ON The HOMOGENEOUS PARTS}

Let $A=\bigoplus_{i \in \mathbb{N}} A_{i}$ be an excellent graded homogeneous Noetherian ring and let $M=\bigoplus_{i \in \mathbb{Z}} M_{i}$ be a finitely generated graded $A$-module. In this section we prove that there is a $k \in \mathbb{N}$, so that for all $n \in \mathbb{N}$ and all $i \geq k$,

$$
U_{C_{n}}^{0}\left(M_{i}\right)=U_{C_{n}}^{0}\left(M_{k}\right) \text { and } U_{S_{n}}^{0}\left(M_{i}\right)=U_{S_{n}}^{0}\left(M_{k}\right),
$$

that is, the codepth and $\left(S_{n}\right)$-loci of the homogeneous parts of $M$ are eventually stable (considered as an $A_{0}$-module). As before we define for all $t \in \mathbb{Z}$

$$
N_{t}=\bigoplus_{i \geq t} M_{i}
$$

and observe the following simple facts: Let $k_{1} \in \mathbb{N}$ be an integer so that for all $t \geq k_{1}, \operatorname{ann}_{A_{0}}\left(M_{t}\right)=\operatorname{ann}_{A_{0}}\left(M_{k_{1}}\right)$. Then for all $t \geq k_{1}$,

$$
U_{C_{n}}^{0}\left(N_{t}\right) \supseteq U_{C_{n}}^{0}\left(N_{k_{1}}\right) \quad \text { and } \quad U_{S_{n}}^{0}\left(N_{t}\right) \supseteq U_{S_{n}}^{0}\left(N_{k_{1}}\right) .
$$

Since $A_{0}$ is Noetherian, there is an integer $k_{2} \in \mathbb{Z}$, so that $k_{2} \geq k_{1}$ and

$$
U_{C_{n}}^{0}\left(N_{t}\right)=U_{C_{n}}^{0}\left(N_{k_{2}}\right) \text { and } U_{S_{n}}^{0}\left(N_{t}\right)=U_{S_{n}}^{0}\left(N_{k_{2}}\right) .
$$

We may also assume for large enough $k_{2}$ that

$$
N_{k_{2}}=A M_{k_{2}}
$$

which implies that for all $t \geq k_{2}$,

$$
N_{t}=A M_{t}
$$

4.1. Lemma. With the assumptions as above assume additionally that $\left(A_{0}, \mathfrak{m}_{0}\right)$ is a local ring. Then there is a $k_{3} \in \mathbb{Z}$, so that for all $t \geq k_{3}$,

$$
\operatorname{depth}_{A_{0}}\left(M_{t}\right)=\operatorname{depth}_{A_{0}}\left(M_{k_{3}}\right)=\operatorname{depth}_{A_{0}}\left(N_{k_{3}}\right) .
$$

Proof. Let $k_{1}$ and $k_{2}$ be as above and take an integer $k$ with $k>k_{2}$. Then $\operatorname{codepth}_{A_{0}}\left(N_{k}\right)=n$ for some $n \in \mathbb{N}$, and therefore

$$
\mathfrak{m}_{0} \in U_{C_{n}}^{0}\left(N_{k}\right) \quad \text { and } \quad \mathfrak{m}_{0} \notin U_{C_{n-1}}^{0}\left(N_{k}\right) .
$$

Since $k \geq k_{2}$, we have for all $t \geq k$

$$
\operatorname{codepth}_{A_{0}}\left(N_{k}\right)=n=\operatorname{codepth}_{A_{0}}\left(N_{t}\right) .
$$

For all $t \geq k_{1}$ we also have that $\operatorname{ann}_{A_{0}}\left(N_{t}\right)=\operatorname{ann}_{A_{0}}\left(N_{k}\right)$, and therefore for all $t \geq k$

$$
\operatorname{depth}_{A_{0}}\left(N_{t}\right)=s=\operatorname{depth}_{A_{0}}\left(N_{k}\right) .
$$

Let $r_{1}, \ldots, r_{s}$ be a maximal regular sequence on $N_{k}$ and put

$$
\bar{N}_{k}=N_{k} /\left(r_{1}, \ldots, r_{s}\right) N_{k} \quad \text { with homogeneous parts } \quad \bar{M}_{i}=M_{i} /\left(r_{1}, \ldots, r_{s}\right) M_{i}
$$

for $i \geq k$. Note that the torsion submodule $\Gamma_{A_{+}}\left(\bar{N}_{k}\right)$ is a finitely generated $A$ submodule of $\bar{N}_{k}$. This implies that there is an integer $k_{3} \geq k$ so that $\Gamma_{A_{+}}\left(\bar{N}_{k}\right) \cap$ $N_{k_{3}}=0=\Gamma_{A_{+}}\left(\bar{N}_{k_{3}}\right)$. Thus for $k_{3}$ large enough the $A$-module $\bar{N}_{k_{3}}$ is $A_{+}$-torsionfree. Since by assumption $\operatorname{depth}_{A_{0}}\left(N_{k}\right)=s=\operatorname{depth}_{A_{0}}\left(N_{k_{3}}\right)$, there is an integer $i \geq k_{3}$ and an element $\bar{x} \in \bar{M}_{i}$ so that $\bar{x} \neq 0$ and $\mathfrak{m}_{0} \bar{x}=0$. Since $\bar{N}_{k_{3}}$ is $A_{+}$-torsionfree, we obtain

$$
\left(A_{+}\right)^{l} \bar{x} \neq 0 \quad \text { for all } \quad l \in \mathbb{N} .
$$


Thus for $k_{4}=i>k_{3}$ we have that $\operatorname{depth}_{A_{0}}\left(\bar{M}_{k_{4}+l}\right)=0$ for all $l \in \mathbb{N}_{0}$, and therefore for all $t \geq k_{4}$,

$$
\operatorname{depth}_{A_{0}}\left(M_{t}\right)=\operatorname{depth}_{A_{0}}\left(M_{k_{4}}\right)=s .
$$

Choose an integer $k_{0} \in \mathbb{Z}$ so that the following conditions are satisfied:

(a) $N_{k_{0}}=A M_{k_{0}}$, that is, $N_{k_{0}}$ is generated in the lowest nonvanishing degree.

(b) For all $t \geq k_{0}, \operatorname{ann}\left(M_{k_{0}}\right)=\operatorname{ann}\left(M_{t}\right)$.

(c) For all $n \in \mathbb{N}_{0}$ and all $t \geq k_{0}$,

$$
U_{C_{n}}^{0}\left(N_{t}\right)=U_{C_{n}}^{0}\left(N_{k_{0}}\right) \text { and } U_{S_{n}}^{0}\left(N_{t}\right)=U_{S_{n}}^{0}\left(N_{k_{0}}\right) .
$$

As before put

$$
Z_{n}=\operatorname{Spec}\left(A_{0}\right) \backslash U_{C_{n}}^{0}\left(N_{k_{0}}\right)=V\left(\mathfrak{b}_{n}\right),
$$

where $\mathfrak{b}_{n} \subseteq A_{0}$ is a reduced ideal. Then $\mathfrak{b}_{n} \subseteq \mathfrak{b}_{n+1}$, yielding an increasing sequence of ideals

$$
\mathfrak{b}_{0} \subseteq \mathfrak{b}_{1} \subseteq \ldots \subseteq \mathfrak{b}_{m-1} \subseteq \ldots
$$

We have seen before that the sequence stops with some $\mathfrak{b}_{m}=A_{0}$, and let $m$ be minimal with this property, that is, let $\mathfrak{b}_{m}=A_{0}$ and $\mathfrak{b}_{m-1} \neq A_{0}$. For all $0 \leq j \leq m-1$ we consider the set of minimal prime divisors of $\mathfrak{b}_{j}$ :

$$
\operatorname{Min}\left(A_{0} / \mathfrak{b}_{j}\right)=\left\{\mathfrak{p}_{j 1}, \ldots, \mathfrak{p}_{j r_{j}}\right\}
$$

By Lemma 4.1, for all $0 \leq j \leq m-1$ and all $r_{j} \geq h \geq 1$, there is an integer $k_{j h} \in \mathbb{N}$ with $k_{j h} \geq k_{0}$, so that for all $i \geq k_{j h}$,

$$
\operatorname{depth}_{\left(A_{0}\right)_{\mathfrak{p}_{j h}}}\left(\left(M_{i}\right)_{\mathfrak{p}_{j h}}\right)=\operatorname{depth}_{\left(A_{0}\right)_{\mathfrak{p}_{j h}}}\left(\left(M_{k_{j h}}\right)_{\mathfrak{p}_{j h}}\right)=\text { constant } .
$$

Let $k=\max \left\{k_{j h} \mid 0 \leq j \leq m-1 ; 1 \leq h \leq r_{j}\right\}$. Then for all $i \geq k$,

$$
\operatorname{depth}_{\left(A_{0}\right)_{\mathfrak{p}_{j h}}}\left(\left(M_{i}\right)_{\mathfrak{p}_{j h}}\right)=\operatorname{depth}_{\left(A_{0}\right)_{\mathfrak{p}_{j h}}}\left(\left(M_{k}\right)_{\mathfrak{p}_{j h}}\right)=\operatorname{depth}_{\left(A_{0}\right)_{\mathfrak{p}_{j h}}}\left(\left(N_{k}\right)_{\mathfrak{p}_{j h}}\right) .
$$

By assumption on the annihilators we also have for all $i \geq k$

$$
\operatorname{dim}_{\left(A_{0}\right)_{\mathfrak{p}_{j h}}}\left(\left(M_{i}\right)_{\mathfrak{p}_{j h}}\right)=\operatorname{dim}_{\left(A_{0}\right)_{\mathfrak{p}_{j h}}}\left(\left(M_{k}\right)_{\mathfrak{p}_{j h}}\right)=\operatorname{dim}_{\left(A_{0}\right)_{\mathfrak{p}_{j h}}}\left(\left(N_{k}\right)_{\mathfrak{p}_{j h}}\right),
$$

which implies that for all $i \geq k$ and all primes $\mathfrak{p}_{j h}$,

$$
\operatorname{codepth}_{\left(A_{0}\right)_{\mathfrak{p}_{j h}}}\left(\left(M_{i}\right)_{\mathfrak{p}_{j h}}\right)=\operatorname{codepth}_{\left(A_{0}\right)_{\mathfrak{p}_{j h}}}\left(\left(M_{k}\right)_{\mathfrak{p}_{j h}}\right)=\operatorname{codepth}_{\left(A_{0}\right)_{\mathfrak{p}_{j h}}}\left(\left(N_{k}\right)_{\mathfrak{p}_{j h}}\right) .
$$

We are now ready to prove:

4.2. Theorem. Let $k$ be as above. Then for all $i \geq k$ and all $\mathfrak{p} \in \operatorname{Spec}\left(A_{0}\right)$,

$$
\operatorname{codepth}_{\left(A_{0}\right)_{\mathfrak{p}}}\left(\left(M_{i}\right)_{\mathfrak{p}}\right)=\operatorname{codepth}_{\left(A_{0}\right)_{\mathfrak{p}}}\left(\left(M_{k}\right)_{\mathfrak{p}}\right) .
$$

Proof. Let $\mathfrak{p} \in \operatorname{Spec}\left(A_{0}\right)$. If $\mathfrak{b}_{0} \nsubseteq \mathbb{p}$, then $\left(N_{k}\right)_{\mathfrak{p}}$ is a Cohen-Macaulay module over $\left(A_{0}\right)_{\mathfrak{p}}$. It follows that $\left(M_{i}\right)_{\mathfrak{p}}$ is Cohen-Macaulay for all $i \geq k$.

Assume that $\mathfrak{b}_{0} \subseteq \mathfrak{p}$ and let $g$ be minimal so that $\mathfrak{b}_{g} \subseteq \mathfrak{p}$ and $\mathfrak{b}_{g+1} \not \mathfrak{p}$. In this case $\operatorname{codepth}_{\left(A_{0}\right)_{\mathfrak{p}}}\left(\left(N_{k}\right)_{\mathfrak{p}}\right)=g+1$, and there is an integer $1 \leq j \leq r_{j}$ so that $\mathfrak{p}_{g j} \subseteq \mathfrak{p}$. By [4, (6.11.5)], the nongraded version of Lemma 2.5, for all $i \geq k$,

$$
\operatorname{codepth}_{\left(A_{0}\right)_{\mathfrak{p}}}\left(\left(M_{i}\right)_{\mathfrak{p}}\right) \geq \operatorname{codepth}_{\left(A_{0}\right)_{p_{g j}}}\left(\left(M_{i}\right)_{p_{g j}}\right)=\operatorname{codepth}_{\left(A_{0}\right)_{\mathfrak{p}_{g j}}}\left(\left(N_{k}\right)_{\mathfrak{p}_{g j}}\right)>g .
$$

In order to verify the other inequality consider

$$
\operatorname{codepth}_{\left(A_{0}\right)_{\mathfrak{p}}}\left(\left(N_{k}\right)_{\mathfrak{p}}\right)=g+1=\operatorname{dim}\left(\left(N_{k}\right)_{\mathfrak{p}}\right)-\operatorname{depth}_{\left(A_{0}\right)_{\mathfrak{p}}}\left(\left(N_{k}\right)_{\mathfrak{p}}\right),
$$


and assume that $\operatorname{depth}_{\left(A_{0}\right)_{\mathfrak{p}}}\left(\left(N_{k}\right)_{\mathfrak{p}}\right)=s$. Let $x_{1}, \ldots, x_{s}$ be a regular sequence on $\left(N_{k}\right)_{\mathfrak{p}}$. Then $x_{1}, \ldots, x_{s}$ is a regular sequence on $\left(M_{i}\right)_{\mathfrak{p}}$ for all $i \geq k$. Since $N_{k}$ and $M_{i}$ have the same annihilators, we obtain that

$$
\operatorname{codepth}_{\left(A_{0}\right)_{\mathfrak{p}}}\left(\left(N_{k}\right)_{\mathfrak{p}}\right)=g+1 \geq \operatorname{codepth}_{\left(A_{0}\right)_{\mathfrak{p}}}\left(\left(M_{i}\right)_{\mathfrak{p}}\right)
$$

for all $i \geq k$. This shows that for all $i \geq k$,

$$
\operatorname{codepth}_{\left(A_{0}\right)_{\mathfrak{p}}}\left(\left(M_{i}\right)_{\mathfrak{p}}\right)=g+1 \text {. }
$$

4.3. Corollary. There is an integer $k \in \mathbb{N}$ so that for all $i \geq k$ and all $n \in \mathbb{N}$,

$$
U_{C_{n}}^{0}\left(M_{i}\right)=U_{C_{n}}^{0}\left(M_{k}\right)=U_{C_{n}}^{0}\left(N_{k}\right) .
$$

4.4. Corollary. There is an integer $k \in \mathbb{N}$ so that for all $i \geq k$ and all $n \in \mathbb{N}$,

$$
U_{S_{n}}^{0}\left(M_{i}\right)=U_{S_{n}}^{0}\left(M_{k}\right)=U_{S_{n}}^{0}\left(N_{k}\right) \text {. }
$$

Proof. The second corollary follows from the first by using Corollary 3.5 .

\section{Applications}

Let $A$ be an excellent ring, let $M$ be a finitely generated $A$-module, and let $I \subseteq A$ be an ideal of $A$. By applying the results of the previous section to the Rees algebra/module and to the associated graded ring/module, respectively, we see that there is an integer $k \in \mathbb{N}$, so that for all $i \geq k$ and all $n \in \mathbb{N}$,

$$
\begin{array}{lll}
U_{C_{n}}\left(I^{i} M\right)=U_{C_{n}}\left(I^{k} M\right) & \text { and } \quad & U_{C_{n}}\left(I^{i} M / I^{i+1} M\right)=U_{C_{n}}\left(I^{k} M / I^{k+1} M\right), \\
U_{S_{n}}\left(I^{i} M\right)=U_{S_{n}}\left(I^{k} M\right) & \text { and } & U_{S_{n}}\left(I^{i} M / I^{i+1} M\right)
\end{array}
$$

In the following we want to apply these results to the $\left(S_{n}\right)$ - and codepth-loci of the modules $M / I^{k} M$. We want to show that these loci are again eventually stable, provided that $M$ is a Cohen-Macaulay module over $A$.

5.1. Lemma. Let $A$ be any Noetherian ring, $I \subseteq A$ an ideal, and $M$ a finitely generated $A$-module. Then for all $k \in \mathbb{N}$,

$$
\operatorname{Supp}\left(M / I^{k} M\right)=\operatorname{Supp}(M / I M) \text {. }
$$

Proof. It suffices to show that for all $k \in \mathbb{N}$,

$$
\operatorname{Supp}\left(M / I^{k} M\right)=\operatorname{Supp}\left(M / I^{k+1} M\right) \text {. }
$$

Since $M / I^{k} M$ is a homomorphic image of $M / I^{k+1} M$, we have $\operatorname{Supp}\left(M / I^{k} M\right) \subseteq$ $\operatorname{Supp}\left(M / I^{k+1} M\right)$. Consider the exact sequence:

$$
0 \rightarrow I^{k} M / I^{k+1} M \rightarrow M / I^{k+1} M \rightarrow M / I^{k} M \rightarrow 0,
$$

and let $\mathfrak{p} \in \operatorname{Spec}(A)$ with $I \subseteq \mathfrak{p}$. The sequence stays exact after localization:

$$
0 \rightarrow\left(I^{k} M / I^{k+1} M\right)_{\mathfrak{p}} \rightarrow\left(M / I^{k+1} M\right)_{\mathfrak{p}} \rightarrow\left(M / I^{k} M\right)_{\mathfrak{p}} \rightarrow 0 .
$$

If $\left(M / I^{k} M\right)_{\mathfrak{p}}=0$ with $\left(M / I^{k+1} M\right)_{\mathfrak{p}} \neq 0$, then

$$
\left(I^{k} M / I^{k+1} M\right)_{\mathfrak{p}}=\left(M / I^{k+1} M\right)_{\mathfrak{p}}
$$

which implies by Nakayama that $\left(M / I^{k+1} M\right)_{\mathfrak{p}}=0$, a contradiction.

A more general version of the next result was proved, using different methods, by Kodiyalam [7, Corollary 9]. 
5.2. Theorem. Suppose that $(A, \mathfrak{m})$ is a local Noetherian ring, let $I \subseteq A$ be an ideal of $A$, and let $M$ be a finitely generated $A$-module. Then there is a $k \in \mathbb{N}$, so that for all $i \geq k$,

$$
\operatorname{depth}_{A}\left(M / I^{i} M\right)=\operatorname{depth}_{A}\left(M / I^{k} M\right) .
$$

Proof. Let $\widehat{A}$ be the $\mathfrak{m}$-adic completion of $A$. Then for any finitely generated $A$ module $T$,

$$
\operatorname{depth}_{A}(T)=\operatorname{depth}_{\widehat{A}}\left(T \otimes_{A} \widehat{A}\right),
$$

and we may replace $A$ by $\widehat{A}$ and $M$ by $M \otimes_{A} \widehat{A}$, and assume that $A$ is excellent. By Lemma 4.1 there is a $k_{1} \in \mathbb{N}$, so that for all $t \geq k_{1}$,

$$
\operatorname{depth}_{A}\left(I^{t} M / I^{t+1} M\right)=\operatorname{depth}_{A}\left(I^{k_{1}} M / I^{k_{1}+1} M\right)=g .
$$

For all $t \geq k_{1}$ consider the exact sequence

$$
0 \rightarrow I^{t} M / I^{t+1} M \rightarrow M / I^{t+1} M \rightarrow M / I^{t} M \rightarrow 0,
$$

which leads to an exact sequence on the cohomology modules:

$$
\begin{gathered}
\cdots \rightarrow H_{\mathfrak{m}}^{i}\left(M / I^{t+1} M\right) \rightarrow H_{\mathfrak{m}}^{i}\left(M / I^{t} M\right) \rightarrow 0 \rightarrow \cdots \rightarrow 0 \\
\rightarrow \cdots \rightarrow H_{\mathfrak{m}}^{g-1}\left(M / I^{t+1} M\right) \rightarrow H_{\mathfrak{m}}^{g-1}\left(M / I^{t} M\right) \rightarrow H_{\mathfrak{m}}^{g}\left(I^{t} M / I^{t+1} M\right) \\
\rightarrow H_{\mathfrak{m}}^{g}\left(M / I^{t+1} M\right) \rightarrow H_{\mathfrak{m}}^{g}\left(M / I^{t} M\right) \rightarrow \cdots,
\end{gathered}
$$

where $g$ is minimal with $H_{\mathfrak{m}}^{g}\left(I^{t} M / I^{t+1} M\right) \neq 0$.

Case 1: There is an $i \leq g-1$ and a $t_{0} \geq k_{1}$, so that $H_{\mathfrak{m}}^{i}\left(M / I^{t_{0}} M\right) \neq 0$. Then for all $t \geq t_{0}, H_{\mathfrak{m}}^{i}\left(M / I^{t} M\right) \neq 0$. Let $h \leq g-1$ be the minimal $i$ with this property. Then

$$
\operatorname{depth}_{A}\left(M / I^{t} M\right)=h \text { for all } t \geq t_{0} .
$$

Case 2: For all $i \leq g-1$ and all $t \geq k_{1}$,

$$
H_{\mathfrak{m}}^{i}\left(M / I^{t} M\right)=0 \text {. }
$$

This implies that $\operatorname{depth}_{A}\left(M / I^{t} M\right) \geq g-1$ for all $t \geq k_{1}$.

Case 2.1: There are infinitely many $t \geq k_{1}$, so that

$$
H_{\mathfrak{m}}^{g-1}\left(M / I^{t} M\right) \neq 0 \text {. }
$$

From the long exact sequence we observe that $H_{\mathfrak{m}}^{g-1}\left(M / I^{t} M\right) \neq 0$ implies that $H_{\mathfrak{m}}^{g-1}\left(M / I^{t-1} M\right) \neq 0$ whenever $t-1 \geq k_{1}$. Thus in this case there is a $t_{1} \geq k_{1}$, so that for all $t \geq t_{1}$,

$$
H_{\mathfrak{m}}^{g-1}\left(M / I^{t} M\right) \neq 0,
$$

and therefore for all $t \geq t_{1}, \operatorname{depth}_{A}\left(M / I^{t} M\right)=g-1$.

Case 2.2: There is a $t_{2} \geq k_{1}$, so that for all $t \geq t_{2}, H_{\mathfrak{m}}^{g-1}\left(M / I^{t} M\right)=0$. Then for all $t \geq t_{2}$,

$$
\operatorname{depth}_{A}\left(M / I^{t} M\right)=g .
$$

5.3. Theorem. Let $A$ be an excellent ring and $M$ a finitely generated CohenMacaulay A-module. Let $I \subseteq A$ be an ideal of $A$ which is not contained in any minimal prime ideal of $M$. Then there is an integer $k \in \mathbb{N}$, so that for all $t \geq k$ and all $n \in \mathbb{N}_{0}$ :

(1) $U_{C_{n}}\left(M / I^{t} M\right)=U_{C_{n}}\left(M / I^{k_{0}} M\right)$.

(2) $U_{S_{n}}\left(M / I^{t} M\right)=U_{S_{n}}\left(M / I^{k_{0}} M\right)$. 
Proof. (1) Fix $n \in \mathbb{N}$ and let $k \in \mathbb{N}$, so that for all $t \geq k$,

$$
U_{C_{n}}\left(I^{t} M\right)=U_{C_{n}}\left(I^{k} M\right) \text {. }
$$

We claim that for all $i \geq k$ and all $\mathfrak{p} \in V(I)$,

$$
\operatorname{depth}_{A_{\mathfrak{p}}}\left(\left(M / I^{i} M\right)_{\mathfrak{p}}\right)=\operatorname{depth}_{A_{\mathfrak{p}}}\left(\left(M / I^{k} M\right)_{\mathfrak{p}}\right) .
$$

Obviously, for all $i \geq k, \operatorname{dim}\left(\left(I^{i} M\right)_{\mathfrak{p}}\right)=\operatorname{dim}\left(\left(I^{k} M\right)_{\mathfrak{p}}\right)$, and thus because of the stability of the codepth-loci, we have for all $\mathfrak{p} \in V(I)$ and all $i \geq k$ that

$$
\operatorname{depth}_{A_{\mathfrak{p}}}\left(\left(I^{i} M\right)_{\mathfrak{p}}\right)=\operatorname{depth}_{A_{\mathfrak{p}}}\left(\left(I^{k} M\right)_{\mathfrak{p}}\right) .
$$

Fix an integer $i \geq k$ and a prime ideal $\mathfrak{p} \in V(I)$, and consider the exact sequence

$$
0 \rightarrow\left(I^{i} M\right)_{\mathfrak{p}} \rightarrow M_{\mathfrak{p}} \rightarrow\left(M / I^{i} M\right)_{\mathfrak{p}} \rightarrow 0 .
$$

With $d=\operatorname{dim}_{A_{\mathfrak{p}}}\left(M_{\mathfrak{p}}\right)=\operatorname{depth}_{A_{\mathfrak{p}}}\left(M_{\mathfrak{p}}\right)$ we obtain a long exact sequence of the local cohomology modules

$$
\begin{gathered}
\cdots \rightarrow 0 \rightarrow H_{\mathfrak{p}}^{i-1}\left(\left(M / I^{i} M\right)_{\mathfrak{p}}\right) \rightarrow H_{\mathfrak{p}}^{i}\left(\left(I^{i} M\right)_{\mathfrak{p}}\right) \rightarrow 0 \rightarrow \cdots \rightarrow 0 \\
\rightarrow H_{\mathfrak{p}}^{d-1}\left(\left(M / I^{i} M\right)_{\mathfrak{p}}\right) \rightarrow H_{\mathfrak{p}}^{d}\left(\left(I^{i} M\right)_{\mathfrak{p}}\right) \rightarrow H_{\mathfrak{p}}^{d}\left(M_{\mathfrak{p}}\right) \rightarrow 0=H_{\mathfrak{p}}^{d}\left(\left(M / I^{i} M\right)_{\mathfrak{p}}\right),
\end{gathered}
$$

where $H_{\mathfrak{p}}^{d}\left(\left(M / I^{i} M\right)_{\mathfrak{p}}\right)=0$, since $\operatorname{dim}_{A_{\mathfrak{p}}}\left(\left(M / I^{i} M\right)_{\mathfrak{p}}\right) \leq d-1$. This shows that

$$
\operatorname{depth}_{A_{\mathfrak{p}}}\left(\left(M / I^{i} M\right)_{\mathfrak{p}}\right)=\operatorname{depth}_{A_{\mathfrak{p}}}\left(\left(I^{i} M\right)_{\mathfrak{p}}\right)-1=\operatorname{depth}_{A_{\mathfrak{p}}}\left(\left(I^{k} M\right)_{\mathfrak{p}}\right)-1
$$

and the claim is proven. For all $i \geq k$ and all $\mathfrak{p} \in V(I)$ we have

$$
\begin{aligned}
\operatorname{depth}_{A_{\mathfrak{p}}}\left(\left(M / I^{i} M\right)_{\mathfrak{p}}\right) & =\operatorname{depth}_{A_{\mathfrak{p}}}\left(\left(M / I^{k} M\right)_{\mathfrak{p}}\right), \\
\operatorname{dim}\left(\left(M / I^{i} M\right)_{\mathfrak{p}}\right) & =\operatorname{dim}\left(\left(M / I^{k} M\right)_{\mathfrak{p}}\right) .
\end{aligned}
$$

The last equation is obtained from Lemma 5.1. This yields that for all $n \in \mathbb{N}$ and for all $i \geq k$,

$$
U_{C_{n}}\left(M / I^{i} M\right)=U_{C_{n}}\left(M / I^{k} M\right) .
$$

The second assumption follows with Corollary 3.5.

5.4. Corollary. Let $A, M$, and $I$ be as in the theorem, and assume that $I M \neq M$. Then there is an element $a \in A$, so that for all $k \in \mathbb{N}$,

(1) $\left(M / I^{k} M\right)_{a} \neq 0$.

(2) $\left(M / I^{k} M\right)_{a}$ is a Cohen-Macaulay module.

5.5. Corollary. Let $A$ be an excellent ring and $M$ a finitely generated A-module. Suppose that the ideal $I \subseteq$ A satisfies the following conditions:

(i) $I$ is not contained in a minimal prime of $M$.

(ii) If $\mathfrak{a} \subseteq A$ is the defining ideal of the non-Cohen-Macaulay locus of $M$, then $\mathfrak{a} \nsubseteq \sqrt{(I M: M)}$.

Then there is an element $a \in A$, so that for all $k \in \mathbb{N}$,

(1) $\left(M / I^{k} M\right)_{a} \neq 0$.

(2) $\left(M / I^{k} M\right)_{a}$ is a Cohen-Macaulay module.

Proof. Choose an element $b \in \mathfrak{a} \backslash \sqrt{(I M: M)}$. In order to prove the assertion apply the previous corollary to the Cohen-Macaulay $A_{b}$-module $M_{b}$. 


\section{REFERENCES}

[1] N. Bourbaki, Commutative algebra, chapters 1-7, Springer Verlag, New York, 1989 MR0979760 (90a:13001)

[2] W. Bruns, J. Herzog, Cohen-Macaulay rings, Cambridge Studies in Advanced Mathematics, Vol. 39, revised edition, Cambridge, 1998 MR1251956 (95h:13020)

[3] H. B. Foxby, Hyperhomological algebra and commutative rings, in preparation

[4] A. Grothendieck, Éléments de géométrie algébrique IV, Inst. Hautes Études Sci. Publ. Math 24 (1965) MR0199181 (33:7330)

[5] M. Hochster, J. L. Roberts, Rings of invariants of reductive groups acting on regular rings are Cohen-Macaulay, Adv. Math. 13 (1974), 115-175 MR0347810 (50:311)

[6] S. Iyengar, Depth for complexes, and intersection theorems, Math. Z. 230 (1999), 545-569 MR.1680036 (2000a:13027)

[7] V. Kodiyalam, Homological invariants of powers of an ideal, Proc. Amer. Math. Soc. 118 (1993), 757-764 MR 1156471 (93i:13022)

[8] H. Matsumura, Commutative ring theory, Cambridge Studies in Advanced Mathematics, Vol. 8, Cambridge, 1986 MR0879273 (88h:13001)

Department of Mathematics, Michigan State University, East Lansing, Michigan 48824

E-mail address: rotthaus@math.msu.edu

Department of Mathematics and Statistics, University of Missouri, Kansas City, Missouri 64110-2499

E-mail address: segal@umkc.edu 